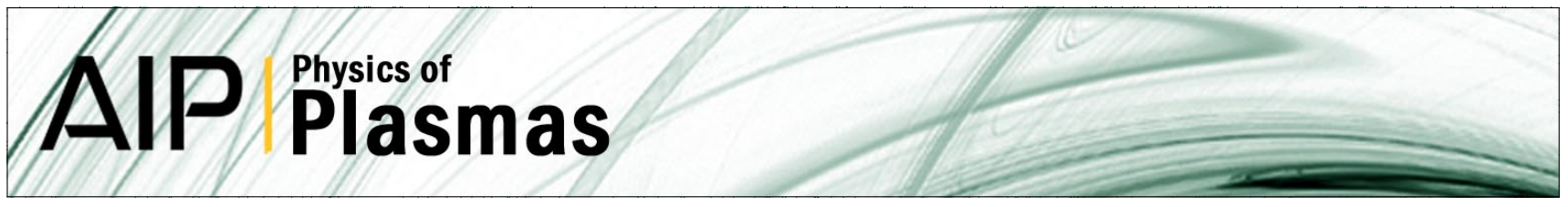

\title{
Relations between light emission and electron density and temperature fluctuations in a helium plasma
}

Shuiliang Ma, John Howard, and Nandika Thapar

Citation: Phys. Plasmas 18, 083301 (2011); doi: 10.1063/1.3620403

View online: http://dx.doi.org/10.1063/1.3620403

View Table of Contents: http://pop.aip.org/resource/1/PHPAEN/v18/i8

Published by the American Institute of Physics.

\section{Related Articles}

Changes in density fluctuations as a result of resonant magnetic perturbations correlate with the density inverse scale length

Phys. Plasmas 19, 024504 (2012)

Poynting vector, energy densities, and pressure of collective transverse electromagnetic fluctuations in unmagnetized plasmas

Phys. Plasmas 19, 012101 (2012)

A generalized flux function for three-dimensional magnetic reconnection

Phys. Plasmas 18, 102118 (2011)

Time-frequency analysis for microwave reflectometry data processing in the HL-2A tokamak

Rev. Sci. Instrum. 82, 103508 (2011)

Fluctuations in collisional plasma in the presence of an external electric field

Phys. Plasmas 18, 102110 (2011)

\section{Additional information on Phys. Plasmas}

Journal Homepage: http://pop.aip.org/

Journal Information: http://pop.aip.org/about/about_the_journal

Top downloads: http://pop.aip.org/features/most_downloaded

Information for Authors: http://pop.aip.org/authors

\section{ADVERTISEMENT}

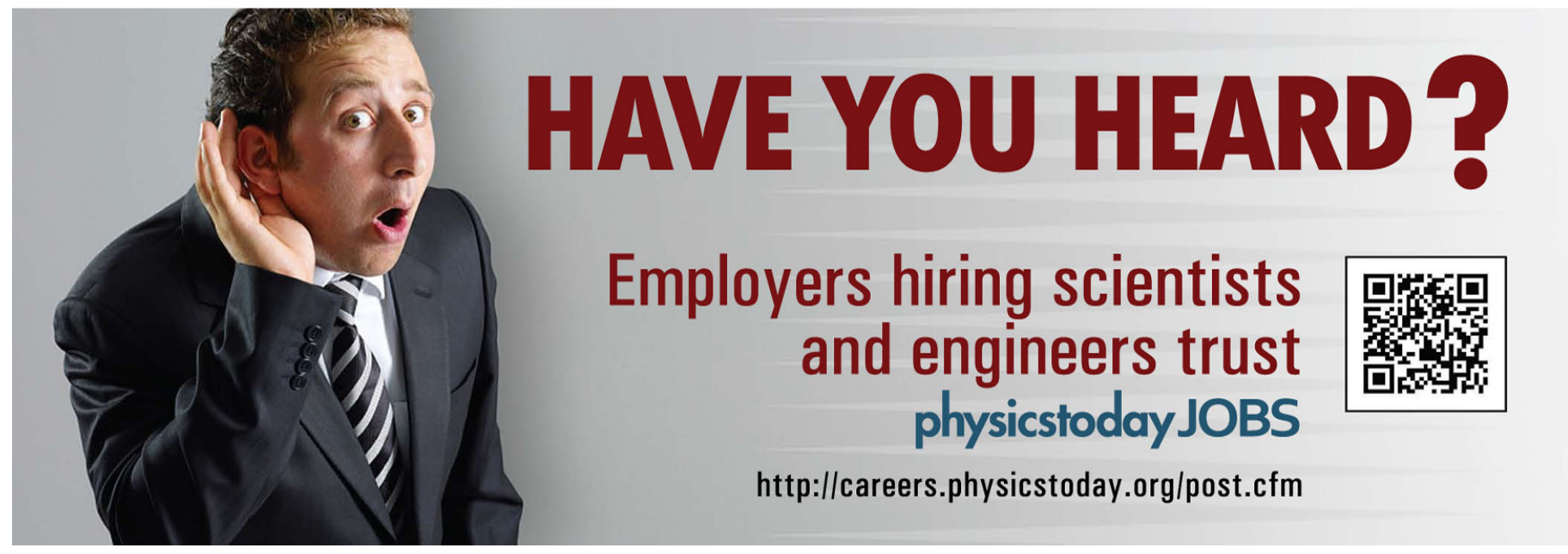




\title{
Relations between light emission and electron density and temperature fluctuations in a helium plasma
}

\author{
Shuiliang Ma, ${ }^{\text {a) }}$ John Howard, and Nandika Thapar \\ Plasma Research Laboratory, Australian National University, Canberra ACT 0200, Australia
}

(Received 28 March 2011; accepted 12 July 2011; published online 11 August 2011)

\begin{abstract}
The relations between three atomic lines, He I $667.8 \mathrm{~nm}\left(3^{1} D \rightarrow 2^{1} P\right), 706.5 \mathrm{~nm}\left(3^{3} S \rightarrow 2^{3} P\right)$, and $728.1 \mathrm{~nm}\left(3^{1} S \rightarrow 2^{1} P\right)$, and the underlying fluctuations in a helium plasma are investigated for the quantitative interpretation of optical observations in plasma fluctuation measurements. Frequency dependent fluctuation amplitude ratios and phase delays between the line emission fluctuation and the electron density and temperature fluctuations are calculated based on a quasi-static collisionalradiative model and a linear approximation technique. For frequencies up to the upper limit of practical interest $(<1 \mathrm{MHz})$, the fluctuation amplitude ratios and phase delays are similar to those directly evaluated by the quasi-static model. It is found that the difference between the results from the linear approximation technique and from the quasi-static model is due to the absence of metastable fluctuations. Contributions of the $2^{1} S$ and $2^{3} S$ metastable fluctuations to the three helium line emission fluctuations are analyzed. The linearity between fluctuations in the line emission and in the electron density and temperature is valid for fluctuation levels higher than 10\%. (C) 2011 American Institute of Physics. [doi:10.1063/1.3620403]
\end{abstract}

\section{INTRODUCTION}

Turbulent plasma fluctuations remain a fundamental and critical issue in magnetically controlled fusion devices. ${ }^{1,2}$ Turbulence, which appears in the form of plasma fluctuations, is thought to be the cause of the anomalous transport in the plasma. The turbulent fluctuations affect fluxes of the particles and energy within the plasma, and thus will deteriorate the confinement. In order to improve the confinement, a better understanding of the physical mechanisms behind the turbulent transport phenomena is important, which requires the plasma fluctuations both in the edge and core of the device to be characterized.

Various diagnostic techniques, such as Langmuir probe, reflectometry, scattering, beam emission spectroscopy, and electron cyclotron emission, have been successfully utilized in different magnetically confined plasmas for the measurement of plasma fluctuations (see Refs. 1-4 for reviews). Among these techniques the optical method is essentially useful, which has the main advantage of a better time and spatial resolution. This method has been widely used for characterizing twodimensional turbulent fluctuations in the edge region of many fusion plasmas by means of beam emission spectroscopy, ${ }^{5-8}$ gas puff imaging, ${ }^{9-13}$ and other optical approaches. ${ }^{14,15}$ The measurements revealed that the edge plasma turbulence often appeared in random structures with long correlation length along the magnetic field and small correlation length across the magnetic field, and sometimes showed intermittent structures moving both poloidally and radially. Also, the plasma fluctuations were always found to have broadband spectrum distributions in the typical frequency range $10 \mathrm{kHz}-1 \mathrm{MHz}$, with relative fluctuation levels increasing monotonically towards the wall. These findings have expanded our knowl-

\footnotetext{
${ }^{a)}$ Electronic mail: shuiliang.ma@anu.edu.au
}

edge about fluctuations and their effects on transport in the edge plasma. Nevertheless, a quantitative characterization of the plasma fluctuation needs the observed light signals to be converted to plasma parameter fluctuations.

This paper aims to explore the connections between the light emission and the plasma parameter fluctuations in a helium plasma. The amount of local plasma line emission, which is determined by basic atomic physical processes within the plasma, is proportional to the population of the upper transition level of the spectral line. Populations in the plasma excited levels can be calculated by using a collisional-radiative (CR) model. In this physical model, the excited level population depends on several plasma parameters, such as the neutral density, the electron density $\left(n_{\mathrm{e}}\right)$ and electron temperature $\left(T_{\mathrm{e}}\right)$. Therefore, any variations in the plasma parameters can be reflected and also possibly be recovered from the changes in the line emission. In recent years, accurate cross section data for neutral helium have been assessed thoroughly. ${ }^{16}$ The model for helium has been widely used for the measurement of $n_{\mathrm{e}}$ and $T_{\mathrm{e}}$ by the line intensity ratio method, ${ }^{17-25}$ which gives consistent results compared with those from other diagnostics. ${ }^{19-22}$ Based on a CR model for neutral helium together with a linear approximation technique, we investigated the relationship between fluctuations in three helium lines, He I $667.8 \mathrm{~nm}\left(3^{1} D \rightarrow\right.$ $\left.2^{1} P\right), 706.5 \mathrm{~nm}\left(3^{3} S \rightarrow 2^{3} P\right)$, and $728.1 \mathrm{~nm}\left(3^{1} S \rightarrow 2^{1} P\right)$, and in the underlying plasma $n_{\mathrm{e}}$ and $T_{\mathrm{e}}$. This relationship is frequency dependent, and thus allows the frequency related fluctuation parameters to be extracted from optical observations, which also provides guidelines for selecting the proper spectral lines to measure the plasma fluctuations. Although the results are only presented for neutral helium plasmas, the general conclusions should be similar for plasmas with other gases. The method presented for helium can be readily used for other gases when an appropriate CR model is available. 
The rest of this paper is organized as follows. In Sec. II we describe the neutral helium CR model for the calculation of excited level populations. Section III proposes a linear approximation technique for the extraction of line emission fluctuation information. Section IV shows the frequency dependent amplitude and phase relationship between the three helium lines and the $n_{\mathrm{e}}$ and $T_{\mathrm{e}}$ fluctuations, as well as influences of two helium metastable states. The applicability of the results for interpretation of optical fluctuation observations is demonstrated by numerical simulations in Sec. V. Limitations and uncertainties of the model are discussed in Sec. VI. Conclusions are drawn in Sec. VII.

\section{HELIUM COLLISIONAL-RADIATIVE MODEL}

\section{A. Population density calculations}

The population densities of excited energy levels in a plasma are determined by the multiple basic physical processes. For helium plasmas at low density $\left(n_{\mathrm{e}}<10^{17} \mathrm{~m}^{-3}\right)$, the population in the excited level can be described by a simple corona model, ${ }^{24-27}$ which only considers the electron impact excitation from the ground state and the spontaneous radiative decay. At higher densities, however, other processes such as excitation from metastable states, collisional excitation and de-excitation, and cascading become important and can no longer be neglected. In this case, a CR model which takes into account many of these processes must be used to accurately describe the excited level populations. In this paper we utilize the widely used CR model for neutral helium developed by Fujimoto ${ }^{28}$ and Goto. ${ }^{29}$

According to the $\mathrm{CR}$ model, the time derivative of the population density of a level $p$ can be described by: $:^{28,29}$

$$
\begin{aligned}
\frac{\mathrm{d} n(p)}{\mathrm{d} t}= & -\left[\sum_{q \neq p} C(p, q) n_{\mathrm{e}}+\sum_{q<p} A(p, q)+S(p) n_{\mathrm{e}}\right] n(p) \\
& +\left[\sum_{q \neq p} C(q, p) n_{\mathrm{e}}+\sum_{q>p} A(q, p)\right] n(q) \\
& +\left[\alpha(p) n_{\mathrm{e}}+\beta(p)+\beta_{\mathrm{d}}(p)\right] n_{\mathrm{e}} n_{\mathrm{i}},
\end{aligned}
$$

where $q$ denotes another energy level, $q<p$ means that level $q$ lies energetically lower than level $p, n_{\mathrm{e}}$, and $n_{\mathrm{i}}$ are densities of the electron and ion, $C(p, q)$ is the electron impact excitation or de-excitation rate coefficient, $A(p, q)$ is the spontaneous transition probability, $S(p)$ is the ionization rate coefficient, and $\alpha(p)$, $\beta(p)$, and $\beta_{\mathrm{d}}(p)$ are the three-body, spontaneous, and dielectric recombination coefficients, respectively. On the right-hand side of the equation, the first set of terms in square brackets represents the population flux out from level $p$, and the second and third sets of terms in square brackets represent the population flux into level $p$. A helium plasma system includes many energy levels and the population density of each level follows Eq. (1). Thus, for a system of $N+1$ levels the coupled ordinary differential equations can be rewritten in the matrix form:

$$
\frac{\mathrm{d} n(p)}{\mathrm{d} t}=\sum_{q=0}^{N} b(p, q) n(q), \quad \mathrm{d} n / \mathrm{d} t=B n,
$$

where $0 \leq p \leq N$, we use $q=0$ to represent the ion energy level, and thus $n(0)=n_{\mathrm{i}}$, and $b(p, q)$ are the elements of the CR matrix $B$, which are functions of $n_{\mathrm{e}}$ and $T_{\mathrm{e}}$.
To simplify the model, the energy levels are generally divided into two sets. ${ }^{30-34}$ One set is time dependent, and the other set can be considered in quasi-static states, i.e., $\mathrm{d} n / \mathrm{d} t \approx 0$. The traditional criterion for dividing the levels into two sets requires that the relaxation times for the excited levels in quasistatic states are much shorter than the relaxation times for the levels in the other set. However, Greenland's analysis ${ }^{33}$ indicates that this requirement is not sufficient. The strict criterion should be based on the knowledge of eigenvectors and eigenvalues of the $\mathrm{CR}$ matrix $B$. Therefore, if the population densities of all the excited levels $s>M$ satisfy the quasi-static approximation, the coupled equations of these levels are easily solved and the population of level $s$, which only depends on the densities of the first $M$ levels and the ion, can be represented by

$$
n(s)=\sum_{q=0}^{M} r(s, q) n(q)
$$

where $r(s, q)$ are the population coefficients for level $s$. Substituting Eq. (3) into (2), one gets the reduced rate equations for levels $p \leq M$ :

$$
\frac{\mathrm{d} n(p)}{\mathrm{d} t}=\sum_{q=0}^{M}\left[b(p, q)+\sum_{s=M+1}^{N} b(p, s) r(s, q)\right] n(q) .
$$

Generally, two CR models have been suggested for neutral helium plasmas. $^{28,29}$ In one model, the set of the timedependent levels consists of only the ground state $\left(1^{1} S\right)$ and the ion $[M=1$ in Eq. (4)]. In the other model, the set of the time-dependent levels also includes the two metastable states $\left(2^{1} S\right.$ and $\left.2^{3} S\right)$ of neutral helium $[M=3$ in Eq. (4)]. Based on Greenland's criteria, Stotler et al. ${ }^{34}$ have investigated the validity of the two models under different plasma conditions. It is found that the $M=3$ model is not always valid though it has a much higher time resolution. Also, under most conditions the initial densities of the two metastables are required for the $M=3$ model. For simplicity, in this paper we only consider the $M=1$ model. In this case, according to Eq. (3) the population densities of the excited levels can be written as

$$
n(s)=r_{0}(s) n_{\mathrm{i}}+r_{1}(s) n_{1} .
$$

Here, $n_{1}$ is the ground state density, and on the right-hand side the first and second terms represent, respectively, the recombining and ionizing plasma components. The rate equations for the ground state and the ion are also simplified:

$$
\frac{\mathrm{d} n_{1}}{\mathrm{~d} t}=-\frac{\mathrm{d} n_{\mathrm{i}}}{\mathrm{d} t}=\alpha_{\mathrm{cr}} n_{\mathrm{e}} n_{\mathrm{i}}-S_{\mathrm{cr}} n_{\mathrm{e}} n_{1}
$$

with $\alpha_{\mathrm{cr}}$ and $S_{\mathrm{cr}}$ representing the CR recombination and ionization rate coefficients, respectively. If all excited energy levels are in quasi-steady state, the population of the excited level can be readily calculated from Eq. (5), provided the densities of the ground state and the ion are known. This is called the quasi-static CR model. If only some of the excited levels can be assumed to be in quasi-steady equilibrium, the time dependent equations for the others are typically rewritten in a form analogous to Eq. (6) [see Eq. (4)] in which the effects of the quasi-static levels are incorporated into 
effective rates. Equation (1) is then reserved those cases in which the quasi-steady assumption is not invoked at all.

For plasmas under different conditions, some physical processes are dominant while others can be neglected. To accurately describe the plasma in a special condition, it is necessary to determine which processes are important. For example, in the edge region of a fusion plasma with high neutral densities the radiation trapping may play an important role, ${ }^{19,20}$ and except in the high density $\left(n_{\mathrm{e}}>10^{20} \mathrm{~m}^{-3}\right)$ and low temperature $\left(T_{\mathrm{e}}<5 \mathrm{eV}\right)$ conditions the recombining plasma component is small and negligible. ${ }^{17}$ The radiation trapping, metastable-metastable collisions and diffusion of metastable atoms are not considered in the code. ${ }^{29}$

\section{B. Quasi-static approximation of excited level populations}

In the calculation of the excited level population densities, since we use the $M=1 \mathrm{CR}$ model the two metastable states and all the excited levels are assumed satisfying the quasi-static approximation. The quasi-static assumption can be checked by using the time dependent CR model (e.g., see Refs. 22, 33-35) in which other important processes affecting the densities can also be included in the CR equations. However, this is highly dependent on the real plasma conditions and requires the details about the plasma. Considering these difficulties, we only consider the simplest case in which other terms, such as transport, sources, and sinks, that affect the ground state density can be neglected. In this case, the quasi-static approximation requires that for ionizing plasmas, the relaxation time of the excited level must be short enough compared with the ionization time of the neutral atoms, so the population in the excited level reaches equilibrium before the variation of neutral density. ${ }^{18}$ By definition, the metastable states have a relatively long relaxation time compared with other excited states. ${ }^{32}$ For neutral helium plasmas, the relaxation time of the $2^{3} S$ metastable is longer than that of the $2^{1} S$ metastable state. ${ }^{23,36}$ This implies that the two metastables, especially the $2^{3} S$ state, are possibly not in equilibrium with the ground state under some plasma conditions.

To check the $2^{3} S$ relaxation time under plasma fluctuations, the temporal evolutions of the excited level populations are calculated with the time dependent CR model. Initially, the ion, the electron, and the ground state densities are specified. The excited state densities are then determined by applying the quasi-static approximation (Eq. (5), same as Eq. (3) with $M=1$ ). The subsequent evolution of the system is obtained by integrating the time dependent CR equations, Eq. (1). Figure 1 shows the temporal evolution of the $2^{3} S$ population normalized to the ground state density, calculated with $T_{\mathrm{e}}=20 \mathrm{eV}$ and several initial $n_{\mathrm{e}}$ values. At $10^{-10}$ s the $n_{\mathrm{e}}$ suddenly increases, leading to the system gradually evolving from the initial steady state to another state. The time at which the population reaches $99 \%$ of the end state value is considered as the relaxation time of the corresponding energy level. The curves show that as the end state $n_{\mathrm{e}}$ increases from $10^{16}$ to $10^{18}$ and $10^{20} \mathrm{~m}^{-3}$, the $2^{3} \mathrm{~S}$ relaxation time decreases from $4 \times 10^{-3}$ to $3 \times 10^{-5}$ and $7 \times 10^{-8} \mathrm{~s}$, respectively. The relaxation time is only dependent on the end state $n_{\mathrm{e}}$ values. The brief flattering of the curve for

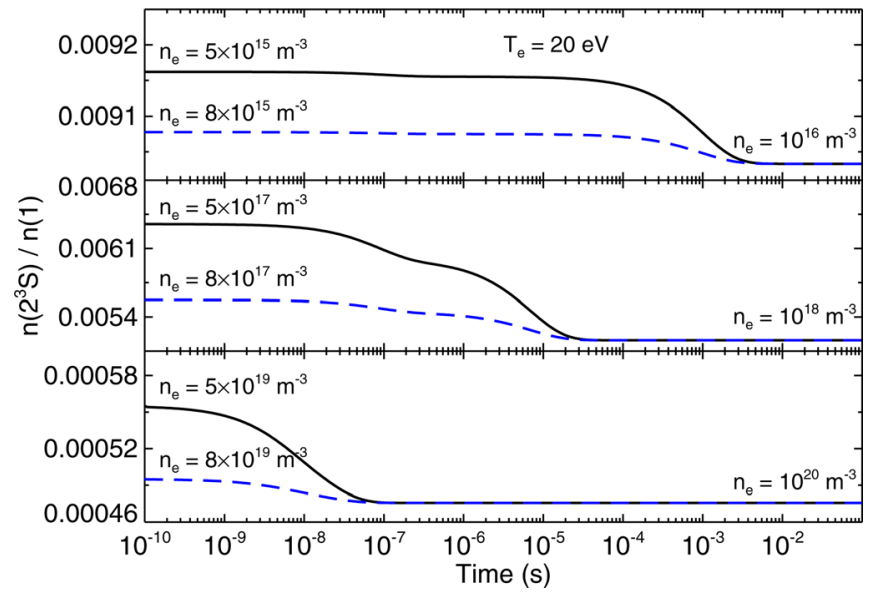

FIG. 1. (Color online) Temporal evolution of the population ratio of the $2^{3} S$ metastable state to the ground state. The $n_{\mathrm{e}}$ at $10^{-10} \mathrm{~s}$ suddenly increases with $20 \%$ and $50 \%$ of the end state values, and the $T_{\mathrm{e}}$ remains $20 \mathrm{eV}$.

$n_{\mathrm{e}}=5 \times 10^{17} \mathrm{~m}^{-3}$ is associated with the relaxation time of the $2^{1} S$ state, indicating a shorter relaxation time compared with that of the $2^{3} S$ state. This effect is also apparent to a lesser degree in the other density curves.

Figures 2(a) and 2(b) show the relaxation time of the $2^{3} S$ metastable (obtained similar as that in Fig. 1) and the ionization time of the ground state (calculated with $1 / S_{\mathrm{cr}} n_{\mathrm{e}}$ ), respectively, as a function of $n_{\mathrm{e}}$ for several $T_{\mathrm{e}}$ values, and Fig. 2(c) shows the ratio between the $2^{3} S$ relaxation time and the ground state ionization time for a comparison. The $2^{3} S$ relaxation time, which is mainly determined by $n_{\mathrm{e}}$ and only weakly dependent on $T_{\mathrm{e}}$, is much smaller than the ground state ionization time at high densities $\left(n_{\mathrm{e}}>10^{18} \mathrm{~m}^{-3}\right)$ and low temperatures $\left(T_{\mathrm{e}}<100 \mathrm{eV}\right)$, as clearly shown in Fig. 2(c). This indicates that the quasi-static approximation is valid for the $2^{3} S$ state under these conditions. For some of the other plasma conditions, however, the relaxation time is comparable with the ionization time. Practically, the ground state density is not determined by the collisional ionization process alone. Other physical processes such as charge exchange, atom-atom collisions, transport of neutral particles, which are ignored in this paper, may also play important roles and partly balance the ionization effect. ${ }^{26}$ Therefore, in real plasmas the characteristic variation time of the ground state density depends on the plasma conditions. This suggests that the quasi-static approximation will possibly still be valid for the $2^{3} S$ state under such plasma conditions.

\section{FLUCTUATION AMPLITUDE RATIO AND PHASE DELAY EXTRACTION METHOD}

The neutral helium line emission and the plasma parameter fluctuations are connected by the basic atomic processes within the plasma. Based on the quasi-static CR model, the variation of the line emissions due to $n_{\mathrm{e}}$ and $T_{\mathrm{e}}$ fluctuations can be directly evaluated. This straightforward method, which is only suitable for low frequency fluctuations, cannot provide any information for plasmas with relatively high fluctuation frequencies. With fluctuations of $n_{\mathrm{e}}$ or $T_{\mathrm{e}}$ in a harmonic form as the inputs to the time dependent CR model, the relationship between the line emission and the plasma 

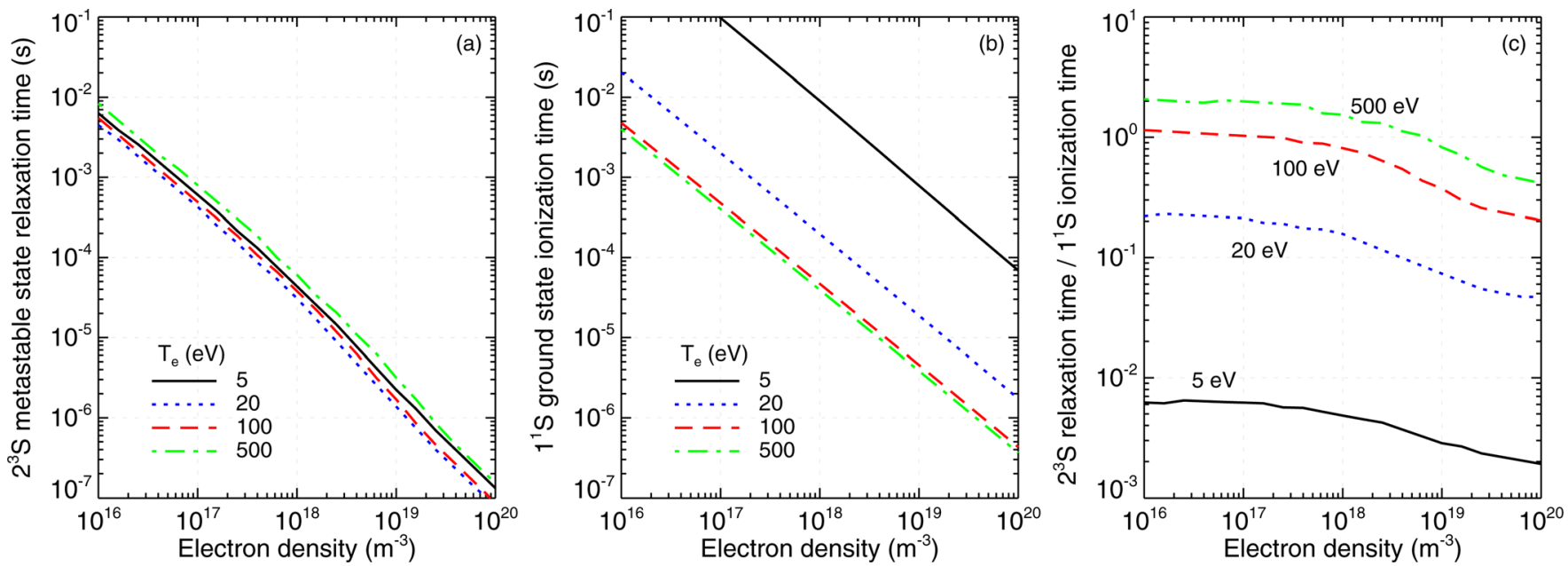

FIG. 2. (Color online) (a) Relaxation time of the $2^{3} \mathrm{~S}$ metastable state, (b) ionization time constant for the ground state calculated with $1 / S_{\mathrm{cr}} n_{\mathrm{e}}$, and (c) the ratio between the $2^{3} \mathrm{~S}$ relaxation time and the ground state ionization time, as a function of $n_{\mathrm{e}}$ for several $T_{\mathrm{e}}$ values. $S_{\mathrm{cr}}$ is the CR ionization coefficient as presented in Eq. (6).

fluctuations as a function of frequency can be obtained. In practice, however, this would be very time consuming for a large number of calculations. Therefore, an alternative method which is more efficient would be desirable.

In this section, we propose a linear approximation method to derive the line emission fluctuation amplitudes and phase delays relative to $n_{\mathrm{e}}$ and $T_{\mathrm{e}}$ fluctuations, which overcomes the above mentioned difficulties. The basic idea of the proposed method is that according to the CR model a low level harmonic fluctuation in $n_{\mathrm{e}}$ or $T_{\mathrm{e}}$ will introduce another harmonic fluctuation in the population of the excited state at the same frequency. Since any fluctuation signals can be approximated by the Fourier series expansion, the variation of the excited state population due to plasma fluctuations can be decomposed in the frequency domain. Therefore, the relationship between fluctuations in the excited state population and in $n_{\mathrm{e}}$ and $T_{\mathrm{e}}$ can be obtained at each fluctuation frequency.

We take the harmonic fluctuations of $n_{\mathrm{e}}$ and $T_{\mathrm{e}}$ at frequency $\omega$ as

$$
\tilde{n}_{\mathrm{e}}(t)=\delta n_{\mathrm{e}} \exp \left[j\left(\omega t+\varphi_{0}\right)\right], \quad \tilde{T}_{\mathrm{e}}(t)=\delta T_{\mathrm{e}} \exp \left[j\left(\omega t+\varphi_{0}\right)\right],
$$

where $\delta n_{\mathrm{e}}$ and $\delta T_{\mathrm{e}}$ are, respectively, the fluctuation amplitudes of $n_{\mathrm{e}}$ and $T_{\mathrm{e}}$ at frequency $\omega$, and $\varphi_{0}$ is the initial phase. The CR matrix $B$ in Eq. (2) is a function of $n_{\mathrm{e}}$ and $T_{\mathrm{e}}$, which can be written to a series expansion in the form of

$$
B\left(n_{\mathrm{e}}, T_{\mathrm{e}}\right)=\sum_{k=0}^{\infty} C_{k}\left(T_{\mathrm{e}}\right) n_{\mathrm{e}}^{k}=\sum_{k=0}^{\infty} D_{k}\left(n_{\mathrm{e}}\right) T_{\mathrm{e}}^{k} .
$$

The temporal variation of the CR matrix due to $n_{\mathrm{e}}$ or $T_{\mathrm{e}}$ fluctuations thus can be expressed with the linear approximation:

$$
\begin{aligned}
\tilde{B}\left(n_{\mathrm{e}}, T_{\mathrm{e}}, t\right) & \approx \frac{\tilde{n}_{\mathrm{e}}(t)}{n_{\mathrm{e}}} \sum_{k=1}^{\infty} k C_{k}\left(T_{\mathrm{e}}\right) n_{\mathrm{e}}^{k}=\frac{\tilde{T}_{\mathrm{e}}(t)}{T_{\mathrm{e}}} \sum_{k=1}^{\infty} k D_{k}\left(n_{\mathrm{e}}\right) T_{\mathrm{e}}^{k} \\
& =\Delta_{B} \exp \left[j\left(\omega t+\varphi_{0}\right)\right] .
\end{aligned}
$$

The truncation value of $k$ in the upper limit of the sum is determined by the accuracy we need. For small perturbations of $n_{\mathrm{e}}$ or $T_{\mathrm{e}}$, the first few order terms can be satisfied.
For relatively low level harmonic fluctuations in $n_{\mathrm{e}}$ or $T_{\mathrm{e}}$, the temporal variation of the excited state population can be approximated by the same harmonic form function but with a different fluctuation amplitude, $\delta n$, and a phase delay, $\varphi$, namely,

$$
\tilde{n}(t)=\delta n \exp \left[j\left(\omega t+\varphi_{0}+\varphi\right)\right] .
$$

By substituting Eqs. (9) and (10) into (2), with $\omega t+$ $\varphi_{0}=0$ and neglecting the higher order terms (with a first order approximation), one obtains

$$
j \omega \delta n e^{j \varphi}=B n+B \delta n e^{j \varphi}+\Delta_{B} n .
$$

Considering the real and imaginary parts of Eq. (11), we get a linear equation system:

$$
\left[\begin{array}{cc}
B & \omega \\
-\omega & B
\end{array}\right]\left[\begin{array}{c}
\delta n \cos \varphi \\
\delta n \sin \varphi
\end{array}\right]=\left[\begin{array}{c}
-B n-\Delta_{B} n \\
0
\end{array}\right] .
$$

For energy levels under quasi-static equilibrium, the term $\mathrm{Bn}$ on the right-hand side should be close to zero and can be neglected. Based on the above equation system, one readily obtains the values of $\delta n \cos \varphi$ and $\delta n \sin \varphi$ by a linear inversion. Then it is straightforward to deduce the population fluctuation amplitude $\delta n$ and phase delay $\varphi$ for each excited level.

It should be noted that $\Delta_{B}$ is a linear function of the fluctuation amplitudes of $n_{\mathrm{e}}$ or $T_{\mathrm{e}}$ as shown in Eq. (9), so under quasi-static condition the fluctuation amplitude $\delta n$ of the excited state population is proportional to the plasma fluctuation levels $\delta n_{\mathrm{e}}$ and $\delta T_{\mathrm{e}}$, with the phase delay value $\varphi$ being unchanged. This is the linear relationship between the line emission fluctuations and the $n_{\mathrm{e}}$ and $T_{\mathrm{e}}$ fluctuations. It is also valuable to note that if we drop the equilibrium condition $B n=0$, the algorithm is applicable to energy levels which are not in quasi-steady states.

In the linear approximation algorithm, the fluctuation amplitude and phase of the ion density is assumed to be the same as those of $n_{\mathrm{e}}$. Both population densities of the $2^{1} S$ and $2^{3} S$ metastable states are considered in quasi-static equilibrium, which thus can be directly computed from Eq. (5). The 


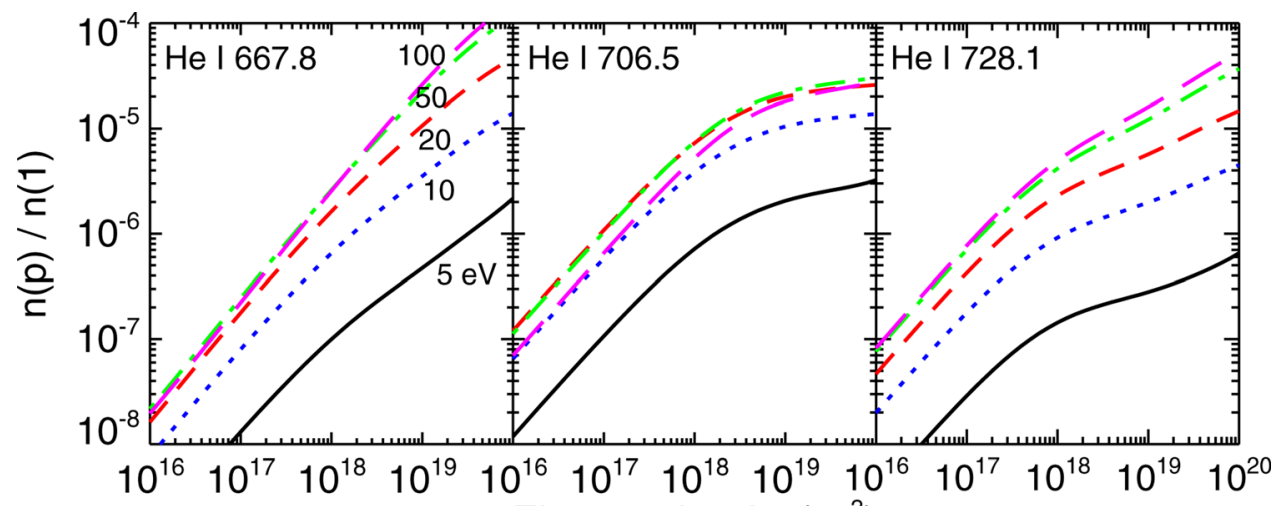

(a)

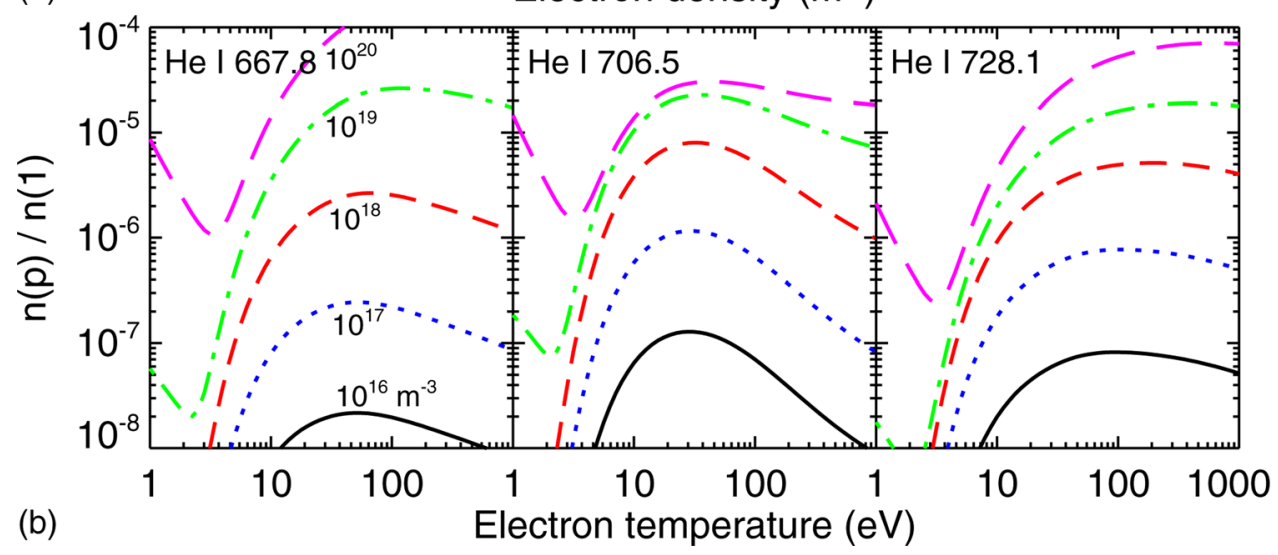

FIG. 3. (Color online) The (a) $n_{\mathrm{e}}$ and (b) $T_{\mathrm{e}}$ dependencies of the population ratio between the upper transition level of the line and the ground state for the three neutral helium lines calculated with $n_{1}=10^{18} \mathrm{~m}^{-3}$. The line emission intensity is proportional to the population ratio as represented by Eq. (13) for ionizing plasmas. ground state is not included in the calculation since the effect of the ground state density fluctuation is found to be not important in some experiments. This will further be discussed in Sec. VI.

\section{RELATIONS BETWEEN LIGHT EMISSION AND $n_{\mathrm{e}}$, $T_{\mathrm{e}}$ FLUCTUATIONS}

\section{A. The $n_{\mathrm{e}}$ and $T_{\mathrm{e}}$ dependencies of spectral line emission}

The CR model for neutral helium presented in Sec. II has been used to calculate the population densities of the excited levels under quasi-static equilibrium, and then to investigate the relationship between spectral line emissions and plasma parameters. Neglecting the recombining plasma component in Eq. (5), the light emission radiated from a level $p$ to a lower level $q$ can be expressed as

$$
I_{p, q}=h c /(4 \pi \lambda) A(p, q) n(p) \propto n_{1} r_{1}\left(n_{\mathrm{e}}, T_{\mathrm{e}}\right),
$$

where $h$ is the Plank's constant, $c$ is the speed of light, and $\lambda$ is the wavelength of the emitted spectral line. Since $I_{p, q}$ is a function of $n_{1}, n_{\mathrm{e}}$, and $T_{\mathrm{e}}$, the variation in the light emission could be due to any fluctuations in the local values of $n_{1}, n_{\mathrm{e}}$, or $T_{\mathrm{e}}$. The $n_{\mathrm{e}}$ and $T_{\mathrm{e}}$ dependencies of the light emission have been discussed for a qualitative explanation of plasma fluctuations from optical observations, ${ }^{9-11,14,37}$ but no extensive study has been presented so far. In this paper, we illustrate the results with the He I 667.8, 706.5, and $728.1 \mathrm{~nm}$ lines as a typical example since among all the neutral helium lines, these three spectral lines are visible and have strong inten- sities, which can be detected in helium or helium seeded plasmas with a wide range of parameters.

Figure 3 shows the light emission of the three helium lines as a function of $n_{\mathrm{e}}$ and $T_{\mathrm{e}}$ calculated by the quasi-static CR model with ground state density $n_{1}=10^{18} \mathrm{~m}^{-3}$ and ion density $n_{\mathrm{i}}=n_{\mathrm{e}}$. The curves represent the population ratios of the upper transition level of the spectral line to the ground state, which are proportional to the corresponding line emission intensity as described by Eq. (13). The $n_{\mathrm{e}}$ and $T_{\mathrm{e}}$ dependencies of the line emission can be easily seen from these curves. In Fig. 3(a), for $n_{\mathrm{e}}<10^{17} \mathrm{~m}^{-3}$ the line emission is almost linearly proportional to $n_{\mathrm{e}}$, since for such relatively low densities the population exclusively excited from the ground state is balanced by the spontaneous radiation decay (the corona model $^{2-27}$ ), i.e., $C(1, p) n_{\mathrm{e}} n_{1}=\sum_{q<p}$ $A(p, q) n(p)$, where $C(1, p)$ is the electron impact excitation rate coefficient from ground state to level $p$. For $n_{\mathrm{e}}>10^{17}$ $\mathrm{m}^{-3}$ the slope of the emission curves decreases due to the increased importance of the secondary physical processes such as excitation and de-excitation from neighboring levels and metastable contributions. In this case, the excited level populations can no longer be approximated by the corona model. In Fig. 3(b), for $T_{\mathrm{e}} \lesssim 25 \mathrm{eV}$ there is a steep increase in the line emission, whereas for higher temperatures the variation of the line emission is relatively small. For $T_{\mathrm{e}} \lesssim 3 \mathrm{eV}$ the decreasing of the line emission with increasing temperature is ascribed to the contribution of the recombining plasma component which cannot be neglected for such low temperatures. The different $T_{\mathrm{e}}$ dependencies of the three helium lines are mainly determined by the electron impact excitation rate coefficients from the ground state which differ 

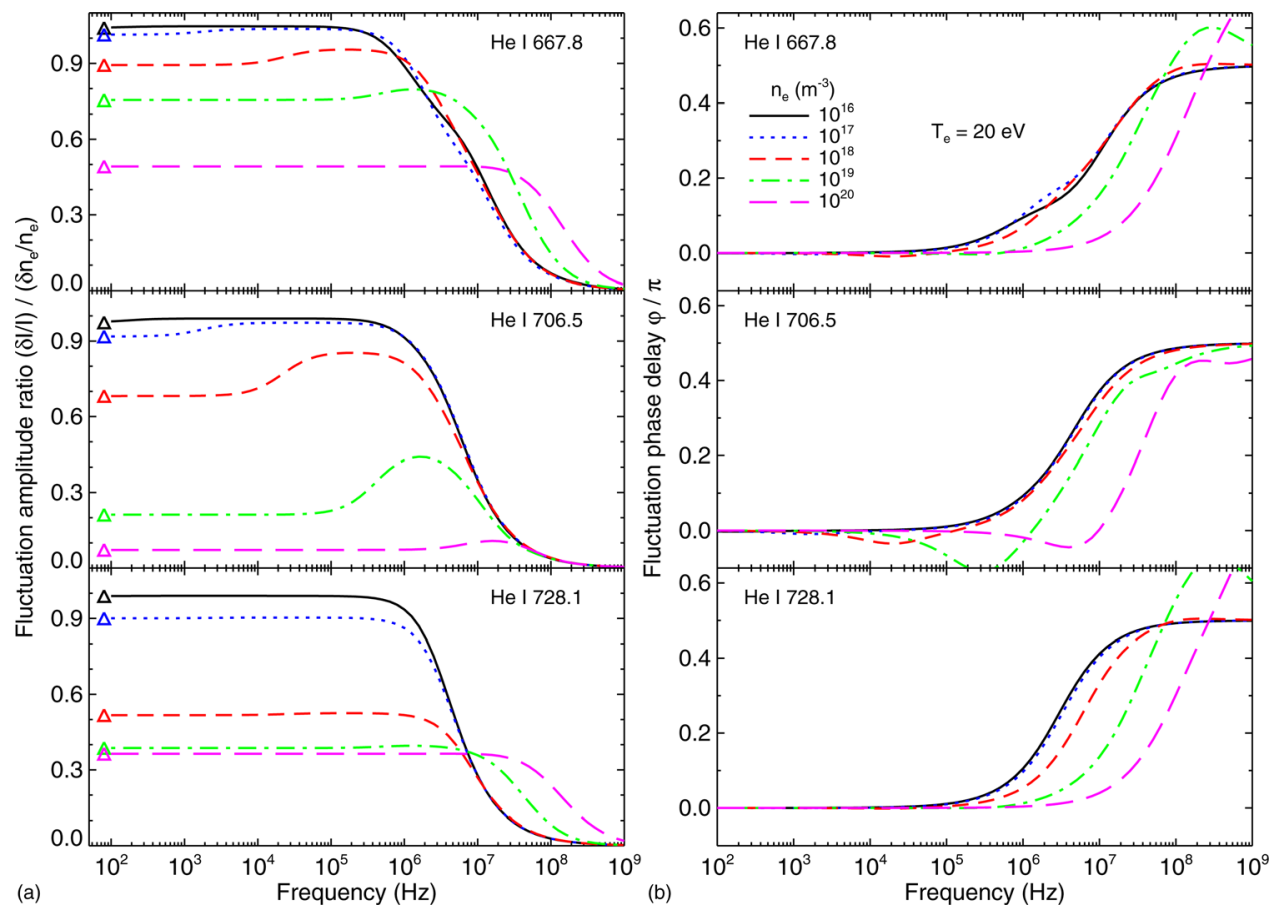

FIG. 4. (Color online) (a) Fluctuation amplitude ratio between the three neutral helium lines and $n_{\mathrm{e}},(\delta I / I) /\left(\delta n_{\mathrm{e}} / n_{\mathrm{e}}\right)$, and (b) the corresponding phase delay, $\varphi / \pi$, as a function of fluctuation frequency for $T_{\mathrm{e}}=20 \mathrm{eV}$ and different $n_{\mathrm{e}}$ values from $10^{16}$ to $10^{20} \mathrm{~m}^{-3}$. The triangular symbols shown in (a) represent the slopes of the $n_{\mathrm{e}}$ dependent emission curves in Fig. 3(a). significantly for the singlet levels and the triplet levels due to the different behavior of cross sections for spin-conserving (singlet excited state) and spin-changing (triplet excited state) collisions. ${ }^{22}$

The absolute value of the emission curve slope is essentially a good estimate of the sensitivity of the line emission to the low level and low frequency plasma fluctuations. As can be seen from Fig. 3, at low density and temperature the line emission is very sensitive to both $n_{\mathrm{e}}$ and $T_{\mathrm{e}}$ fluctuations while for relatively high density and temperature values the sensitivity to $n_{\mathrm{e}}$ fluctuation decreases and the sensitivity to $T_{\mathrm{e}}$ fluctuation becomes very low especially for the singlet spectral lines. Although based on the quasi-static CR model results the relationship between the fluctuations in the line emission and in $n_{\mathrm{e}}$ and $T_{\mathrm{e}}$ is clear, the relationship as a function of frequency is still not clear. The frequency dependent information is important for the quantitative interpretation of optical measurement of $n_{\mathrm{e}}$ and $T_{\mathrm{e}}$ fluctuations, because in fusion plasmas the fluctuations have been found to have a broadband frequency spectrum (e.g., see Refs. 2-4). We therefore show in the following Secs. IV B and IV $\mathrm{C}$ the results obtained by the linear approximation technique described in Sec. III.

\section{B. Electron density caused fluctuations}

According to Eq. (13), the fluctuation of the spectral line emission could be caused by variations in $n_{\mathrm{e}}$ of the plasma. The relationship between the line emission and $n_{\mathrm{e}}$ fluctuation is obtained as follows. First, the excited population densities at a given $n_{\mathrm{e}}$ and $T_{\mathrm{e}}$ are calculated from the $M=1$ quasi-static CR mode, Eq. (5). Then, the fluctuation amplitude and phase delay for each state are determined by solving Eq. (12) with $\Delta_{B}$ being obtained from imposed $n_{\mathrm{e}}$ fluctuation based on Eq. (9) (noting that $B n=0$ since the quasi-static approximation is used for the calculation of the excited state population densities). Figure 4 shows the fluctuation amplitude ratio and phase delay profiles for the three helium lines as a function of frequency calculated with $T_{\mathrm{e}}=20 \mathrm{eV}$ and several $n_{\mathrm{e}}$ values. The profiles in Fig. 4(a) show that at relatively low frequencies the ratio is flat, i.e., it is independent of the fluctuation frequency. As the frequency increases, the ratio first increases slightly and then stays constant, which is especially evident for the He I $706.5 \mathrm{~nm}$ line. A further increase in the frequency leads to the ratio sharply decreasing to zero. The phase delay profiles shown in Fig. 4(b) increase from zero at low frequencies to $\pi / 2$ at very high frequencies. For the phase delay profiles, there are also changes corresponding to the variations in the amplitude ratio profiles: where the ratio increases the phase delay becomes negative and the decrease in the ratio is always associated with the increase in the phase delay.

Figure 4 also shows the fluctuation amplitude ratio and phase delay profiles for different $n_{\mathrm{e}}$ values. As $n_{\mathrm{e}}$ increases, the ratio decreases, the "hump" (see Fig. 4(a), which is most evident for the He I $706.5 \mathrm{~nm}$ line) moves to higher fluctuation frequencies, and the frequency where the ratio begins to decrease or where the phase delay begins to increase becomes higher. In Fig. 4(a), the data denoted by the triangular symbols are the slopes of the curves shown in Fig. 3(a), for a comparison with the values calculated by the linear approximation method. The identical results at low frequencies indicate that all the excited levels including the two metastables are in equilibrium with the plasma density fluctuation, and thus there is no phase delay between the fluctuation of the excited level population and $n_{\mathrm{e}}$ fluctuation. The comparison also indicates that the "hump" in Fig. 4(a), more exactly speaking, the difference between the amplitude ratios at low frequencies and those in the "hump" range is due to the falling out of equilibrium of some energy levels. In fact, the "hump" and the negative phase delays are the results of the absence of the metastable population fluctuations, which will be discussed in detail in Sec. IV D. The sharp 
decrease in the amplitude ratio at very high frequencies is due to the limited relaxation time of the upper transition level of the spectral line; for frequencies higher than the value corresponding to the relaxation time, the population in the excited level cannot attain equilibrium speedily or alternatively has no response to the fluctuations. The excited level relaxation time is evident from the decrease in the ratio profiles: as $n_{\mathrm{e}}$ increases from $10^{16}$ to $10^{20} \mathrm{~m}^{-3}$ the relaxation time of the upper transition levels of the three helium lines decreases from $\sim 10^{-6}$ to $\sim 2 \times 10^{-8}$ s. Even under the lowest plasma density condition, the excited level relaxation time still corresponds to a frequency higher than $10^{6} \mathrm{~Hz}$, thus the optical method is applicable for the measurement of plasma fluctuations at any practically interested frequencies.

The same calculation was performed for other $n_{\mathrm{e}}$ and $T_{\mathrm{e}}$ values under $n_{\mathrm{e}}$ fluctuations. Considering that the fluctuation amplitude ratio and phase delay between the line emissions and the $n_{\mathrm{e}}$ fluctuation at relatively low frequencies are almost flat and close to zero, respectively, we only presented the results of the fluctuation ratios at frequency $10^{3} \mathrm{~Hz}$, as shown in Fig. 5. One can see that the ratio is almost uniform at different densities and temperatures for the $\mathrm{He} \mathrm{I} 667.8 \mathrm{~nm}$ line, and has a weak $T_{\mathrm{e}}$ dependence and a strong $n_{\mathrm{e}}$ dependence for both the He I 706.5 and $728.1 \mathrm{~nm}$ lines. At low $n_{\mathrm{e}}$ values the ratio is close to 1 as in this range the emission is proportional to $n_{\mathrm{e}}$ (the corona model). As $n_{\mathrm{e}}$ increases the sensitivity of the line emission to $n_{\mathrm{e}}$ fluctuation decreases from about 1 to 0.6 , 0.1 , and 0.3 , respectively, for the He I 667.8, 706.5, and 728.1 $\mathrm{nm}$ lines. The decreasing of the fluctuation ratio with increasing $n_{\mathrm{e}}$ is due to the change of the population mechanisms of the excited states, as already mentioned in Sec. IV A.

\section{Electron temperature caused fluctuations}

According to Eq. (13), the fluctuation of the spectral line emission could also be caused by variations in $T_{\mathrm{e}}$ of the

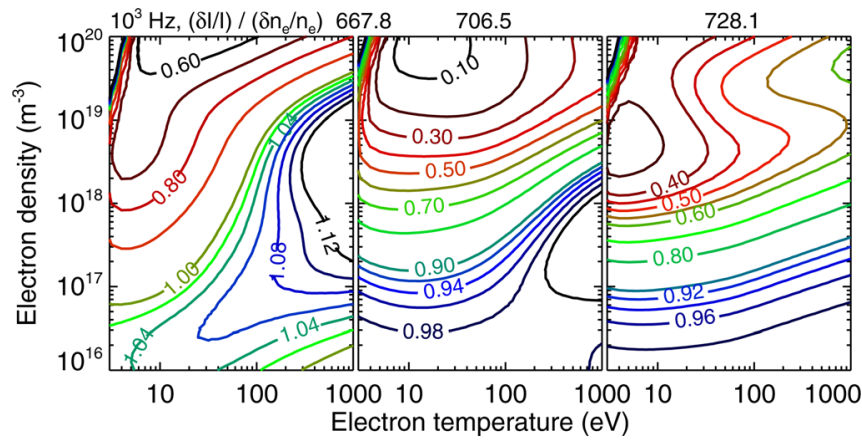

FIG. 5. (Color online) Contour plot of the relative fluctuation amplitude ratio for the three neutral helium lines due to $n_{\mathrm{e}}$ fluctuations, $(\delta I / I) /\left(\delta n_{\mathrm{e}} / n_{\mathrm{e}}\right)$, as a function of $n_{\mathrm{e}}$ and $T_{\mathrm{e}}$ at $10^{3} \mathrm{~Hz}$.

plasma. The $T_{\mathrm{e}}$ related fluctuations of the helium line emission were investigated in a similar way as those of the $n_{\mathrm{e}}$ related fluctuations. Figure 6 shows the fluctuation amplitude ratio and phase delay profiles due to $T_{\mathrm{e}}$ fluctuation as a function of frequency calculated with $n_{\mathrm{e}}=5 \times 10^{17} \mathrm{~m}^{-3}$ and several $T_{\mathrm{e}}$ values. The results are almost the same as those of $n_{\mathrm{e}}$ fluctuation. For frequencies lower than $\sim 3 \times 10^{6} \mathrm{~Hz}$, the ratio is nearly independent of fluctuation frequency and the phase delay is close to zero or $-\pi$, except for frequencies close to $10^{4} \mathrm{~Hz}$, where there are minor variations (under most plasma parameters a decrease) in the ratio profiles and the phase delay becomes slightly higher than zero or $-\pi$. These changes are also due to the metastable population fluctuations which gradually disappear as frequency increases (more details are presented in Sec. IV D). For frequencies higher than $\sim 3 \times 10^{6} \mathrm{~Hz}$, the sensitivity of the line emission fluctuation to $T_{\mathrm{e}}$ fluctuation decreases sharply to zero and the corresponding phase delay increases to $\pi / 2$ or $-\pi / 2$, which are also due to the limited relaxation time of the spectral line upper transition level. The relaxation time is almost independent of $T_{\mathrm{e}}$, it is only a strong function of $n_{\mathrm{e}}$. Hence, the
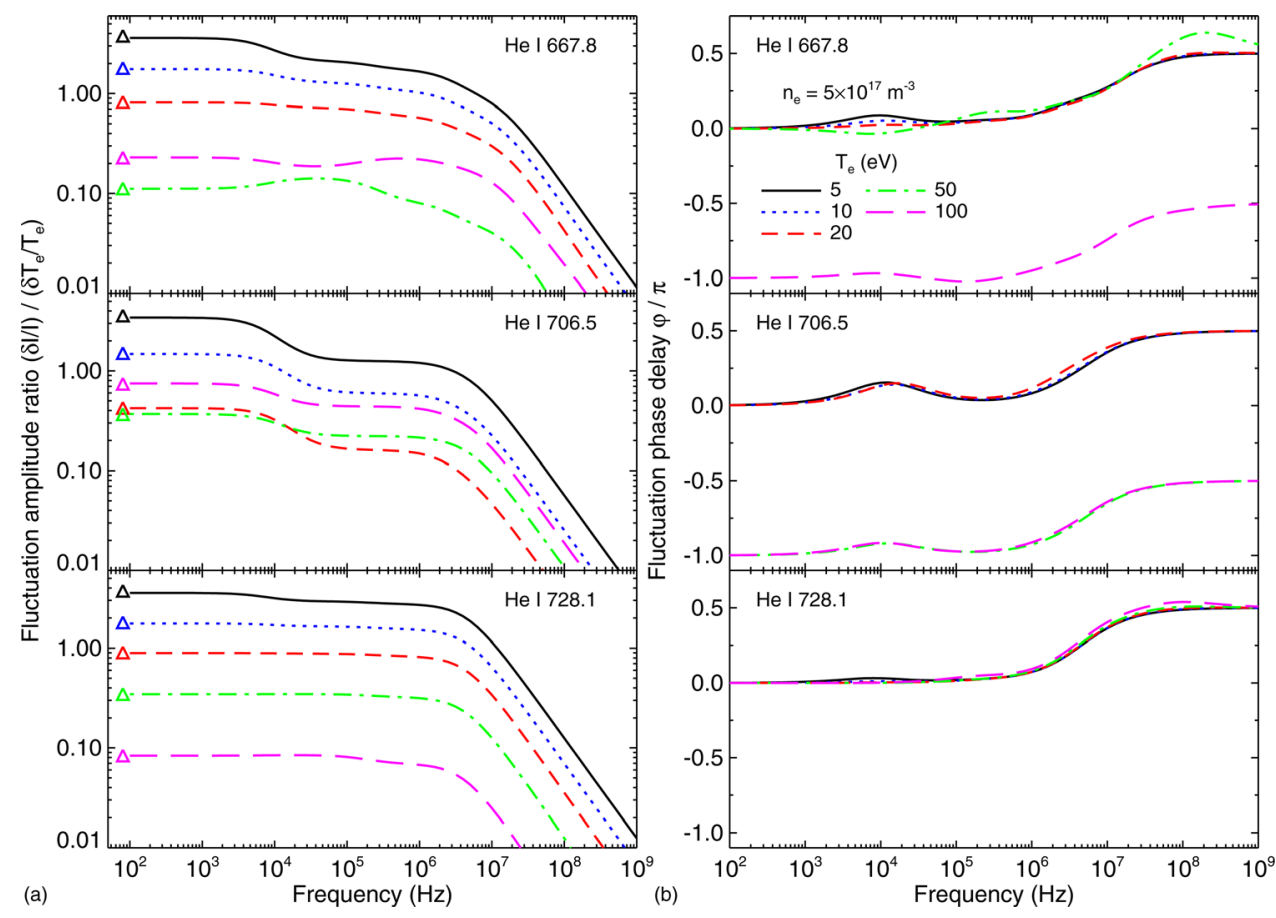

FIG. 6. (Color online) (a) Fluctuation amplitude ratio between the three neutral helium lines and $T_{\mathrm{e}},(\delta I / I) /\left(\delta T_{\mathrm{e}} / T_{\mathrm{e}}\right)$, and (b) the corresponding phase delay, $\varphi / \pi$, as a function of fluctuation frequency for $n_{\mathrm{e}}=5 \times 10^{17} \mathrm{~m}^{-3}$ and different $T_{\mathrm{e}}$ values from 5 to $100 \mathrm{eV}$. The triangular symbols shown in (a) represent the slopes of the $T_{\mathrm{e}}$ dependent emission curves in Fig. 3(b). 
fluctuation ratio profiles for different temperatures start to decrease at about the same frequency.

In Fig. 6(a), the data represented by the triangular symbols are the slopes of the $T_{\mathrm{e}}$ dependent curves shown in Fig. 3(b) at several $T_{\mathrm{e}}$ values, which are in good agreement with the fluctuation amplitude ratios between the line emission and $T_{\mathrm{e}}$ at low frequencies, calculated by the linear approximation method. The ratio decreases with increasing $T_{\mathrm{e}}$ values. But for the He I 667.8 and $706.5 \mathrm{~nm}$ lines, the ratio increases for $T_{\mathrm{e}}$ higher than 50 and $20 \mathrm{eV}$, respectively, and the corresponding phase values are reversed (with a $\pi$ difference). This phenomenon can be explained based on the line emission profiles as a function of $T_{\mathrm{e}}$ shown in Fig. 3(b). For $T_{\mathrm{e}} \lesssim 25 \mathrm{eV}$ there is a sharp increase in the line emission since the excitation energy from the ground state to the excited levels is $\sim 23 \mathrm{eV}$, and for $T_{\mathrm{e}}>25 \mathrm{eV}$ the line emission first reaches a maximum and then decreases gradually. The absolute value of the curve slope (equivalent to the fluctuation amplitude ratio at low frequencies) thus first decreases to zero and then increases gradually. The reversal of the excited level fluctuation phase can also be explained according to the relationship between the line emission and $T_{\mathrm{e}}$. For example, the emission profile of the $706.5 \mathrm{~nm}$ line has a decreasing trend for $T_{\mathrm{e}} \gtrsim 30 \mathrm{eV}$, thus an increase in $T_{\mathrm{e}}$ will lead to a decrease in the line emission and vice versa. Hence, the phase of the emission fluctuation is reversed compared with that of the $T_{\mathrm{e}}$ fluctuation.

The same calculation was performed for other $n_{\mathrm{e}}$ and $T_{\mathrm{e}}$ values under $T_{\mathrm{e}}$ fluctuations. Figure 7 shows the fluctuation ratios for the line emissions at the frequency of $10^{3} \mathrm{~Hz}$ due to $T_{\mathrm{e}}$ fluctuation. As expected, the ratio is only a weak function of $n_{\mathrm{e}}$ but a strong function of $T_{\mathrm{e}}$. The sensitivity of the line emission fluctuation to $T_{\mathrm{e}}$ fluctuation is strong at low temperatures (with a maximum fluctuation ratio of $\sim 5$ ), while there is almost no response for the He I 667.8, 706.5, and $728.1 \mathrm{~nm}$ lines at temperatures of 50,30 , and $100 \mathrm{eV}$, respectively. At higher temperatures the fluctuation ratio has a slight gradual increase. This variation trend has already been discussed in Sec. IV A, as shown in Fig. 3(b) due to the $T_{\mathrm{e}}$ dependencies of the electron impact excitation rate coefficients from the ground state to the upper transition level of the three helium lines.

It should be noted that the fraction of the line emission fluctuation caused by $T_{\mathrm{e}}$ fluctuations is relatively small at

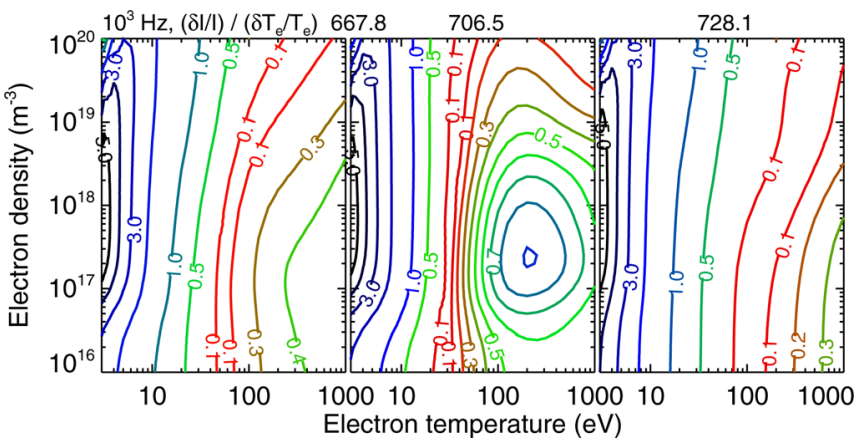

FIG. 7. (Color online) Contour plot of the relative fluctuation amplitude ratio for the three helium lines due to $T_{\mathrm{e}}$ fluctuations, $(\delta I / I) /\left(\delta T_{\mathrm{e}} / T_{\mathrm{e}}\right)$, as a function of $n_{\mathrm{e}}$ and $T_{\mathrm{e}}$ at $10^{3} \mathrm{~Hz}$. high temperatures, especially for the singlet spectral lines. In some temperature ranges, there is almost no fluctuation for the line emission. The insensitive regions for the three helium lines cover a temperature interval from $\sim 20$ to $\sim 500 \mathrm{eV}$. In these regions the measured line emission essentially reflects the $n_{\mathrm{e}}$ fluctuations, since the variation in the line emission due to $T_{\mathrm{e}}$ fluctuations is negligibly small. This is possibly one of the reasons why in many experiments the observed optical fluctuations have similar frequency spectra and spatial structures compared with the probe measured saturated ion current fluctuations, ${ }^{5,11,14,15}$ remembering that the fluctuation ratio between the line emission and $n_{\mathrm{e}}$ is almost independent on frequency. In these $T_{\mathrm{e}}$ fluctuation insensitive regions the $n_{\mathrm{e}}$ fluctuation can be directly recovered from the signal of only one neutral helium line emission, provided $n_{\mathrm{e}}$ and $T_{\mathrm{e}}$ are measured by other methods. On the other hand, we have also to note that in some other regions, for example $n_{\mathrm{e}}>10^{19} \mathrm{~m}^{3}$ and $30<T_{\mathrm{e}}<80 \mathrm{eV}$, the He I $706.5 \mathrm{~nm}$ line is not sensitive to either $n_{\mathrm{e}}$ or $T_{\mathrm{e}}$ variations, thus it is not suitable for plasma fluctuation measurements.

\section{Contribution of metastable population fluctuations}

As noted in Secs. IV B and IV C, the fluctuations of the ${ }^{1} S$ and $2^{3} S$ metastables will cause some influences on the fluctuation of the excited level population. To further check this issue and determine to what extent the metastable population fluctuations should be considered, the fractions of the two metastable contributions to the helium spectral line fluctuations were investigated. In the linear approximation algorithm [Eq. (12)], any energy level can be excluded, i.e., the level is considered using the quasi-static population without fluctuations. The difference between the results obtained with the level being included and excluded represents the population fluctuation contribution from this level. In this way, the contributions of the metastable fluctuations and other contributions to the excited level population fluctuation can be evaluated.

As a typical example, Fig. 8 shows the contributions of the two metastables and other contributions to the three neutral helium line emission fluctuations as a function of frequency. The excited level populations were calculated under the quasi-static condition with $n_{\mathrm{e}}=5 \times 10^{17} \mathrm{~m}^{-3}$ and $T_{\mathrm{e}}=20 \mathrm{eV}$. The total contribution was obtained same as that shown in Figs. 4 and 6 . The $2^{1} S, 2^{3} S$, and other contributions were then determined by comparing the total contribution and the results calculated with either one or both the $2^{1} S$ and $2^{3} S$ metastables being excluded in the linear approximation algorithm. In the case of $n_{\mathrm{e}}$ fluctuation [Fig. 8(a)], the contribution of the $2^{1} S$ state is very small for the three neutral helium lines and can be completely neglected, while the contribution of the $2^{3} S$ state is relatively large for the He I 667.8 and $706.5 \mathrm{~nm}$ lines, $\sim 10 \%$ and $\sim 40 \%$, respectively, and small for the He I $728.1 \mathrm{~nm}$ line. In the case of $T_{\mathrm{e}}$ fluctuation [Fig. 8(b)], the $2^{1} \mathrm{~S}$ contribution becomes important for the He I $667.8 \mathrm{~nm}$ line, while the $2^{3} S$ contribution is still significant for both the He I 667.8 and $706.5 \mathrm{~nm}$ lines, $\sim 10 \%$ and $\sim 54 \%$, respectively. The He I $728.1 \mathrm{~nm}$ line shows almost no sensitivity to any of the two metastables. From the 


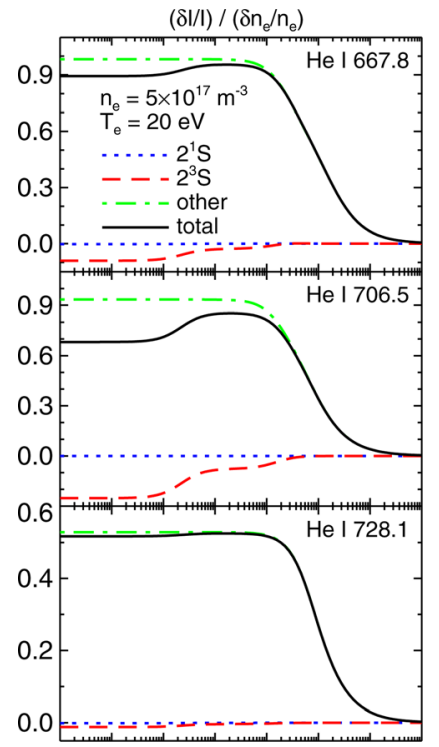

$\begin{array}{llllllll}10^{2} & 10^{3} & 10^{4} & 10^{5} & 10^{6} & 10^{7} & 10^{8} & 10^{9}\end{array}$ (a)

Frequency $(\mathrm{Hz})$ $(\delta / /) /\left(\delta \mathrm{T}_{\mathrm{e}} / \mathrm{T}_{\mathrm{e}}\right)$
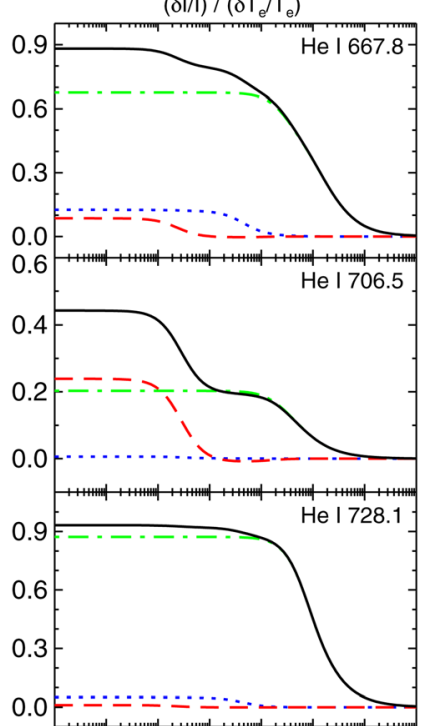

$10^{2} 10^{3} 10^{4} \quad 10^{5} 10^{6} 10^{7} 10^{8} 10^{9}$ (b)

FIG. 8. (Color online) Contributions of the $2^{1} S$ and $2{ }^{3} S$ metastable states population fluctuations and other contributions to the three neutral helium line emission fluctuations as a function of frequency in the case of (a) $n_{\mathrm{e}}$ and (b) $T_{\mathrm{e}}$ fluctuations, calculated with $n_{\mathrm{e}}=5 \times 10^{17} \mathrm{~m}^{-3}$ and $T_{\mathrm{e}}=20 \mathrm{eV}$.

profiles one can also see that under the current plasma conditions, the relaxation time of the $2^{3} S$ state is $\sim 7 \times 10^{-5} \mathrm{~s}$ compared with $\sim 3 \times 10^{-6}$ s of the $2^{1} S$ state, which are clearly indicated by the gradual decreasing of the metastable contribution profiles with increasing frequency as in Fig. 8(b) for the He I $667.8 \mathrm{~nm}$ line. The relaxation time indicated

by the variation of these profiles agrees with the results shown in Fig. 2(a).

In Fig. 8(a), the inclusion of the metastable fluctuations decreases the excited level population fluctuations. This indicates the fluctuation of the $2^{3} S$ metastable has a reversed phase compared with the $n_{\mathrm{e}}$ fluctuation, noticing that the excited level population fluctuations are in phase with $n_{\mathrm{e}}$ fluctuation as shown in Fig. 4(b). One can clearly see from Fig. 1 that the $2^{3} S$ population decreases with increasing $n_{\mathrm{e}}$, which confirms the reversed $2^{3} S$ population fluctuation. As fluctuation frequency increases, the phase delay between the $2^{3} S$ state and $n_{\mathrm{e}}$ increases. This leads to a negative phase delay in the excited levels. A further increase in the frequency will cause a larger phase delay for the $2^{3} S$ state, but at the same time the contribution from this metastable becomes negligible (because the metastable has almost no response to $n_{\mathrm{e}}$ fluctuation). The net result is that the $2^{3} S$ phase delay has little effect on the excited level for which the phase delay returns to zero. Hence, in Fig. 4 where the fluctuation ratio increases the corresponding phase delay first decreases to below zero and then gradually increases back to zero. In Fig. 8(b), the inclusion of the metastable fluctuations enhances the excited level population fluctuations. This indicates that the metastable fluctuations are in phase with $T_{\mathrm{e}}$ fluctuations. The variation of the phase delay profiles in Fig. 6(b) corresponding to the decreasing of the fluctuation ratio in Fig. 6(a) near the frequency $10^{4}$ $\mathrm{Hz}$ can be explained in a similar manner.

To determine in which plasma parameter region the contributions from the two metastables are important, contributions to the three helium lines were calculated at different $n_{\mathrm{e}}$ and $T_{\mathrm{e}}$ values. Figure 9 shows the $2^{1} S$ and $2{ }^{3} S$ metastable

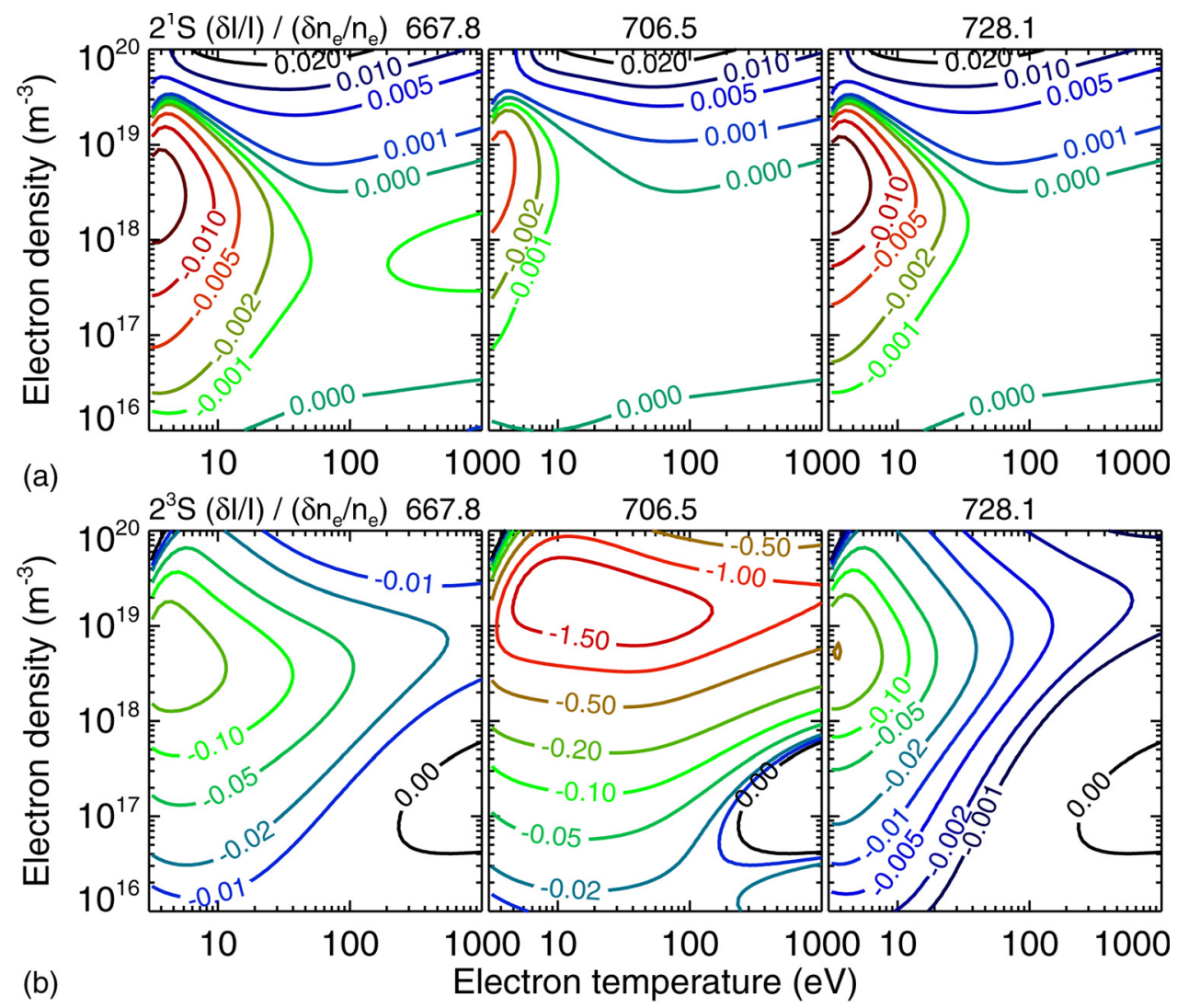

FIG. 9. (Color online) Contributions of the (a) $2^{1} S$ and (b) $2^{3} S$ metastable states population fluctuations to the three neutral helium line emission fluctuations in the $n_{\mathrm{e}}$ fluctuation related component at $10^{3} \mathrm{~Hz}$. 
contributions due to $n_{\mathrm{e}}$ fluctuation calculated at frequency $10^{3} \mathrm{~Hz}$. It is shown that the $2^{1} S$ contribution is negligibly low $(<2 \%)$ for all the three atomic lines, while the $2^{3} S$ contribution for the He I 667.8 and $728.1 \mathrm{~nm}$ lines is relatively large only in a small region where $5 \times 10^{17}<n_{\mathrm{e}}<4 \times 10^{19}$ $\mathrm{m}^{-3}$ and $T_{\mathrm{e}}<20 \mathrm{eV}$, in other regions the contribution is far less than $10 \%$. The $2^{3} S$ contribution for the He I $706.5 \mathrm{~nm}$ line becomes important at $n_{\mathrm{e}}>10^{17} \mathrm{~m}^{-3}$, in the lower density regions the contribution is small because the metastable population is less likely being changed by $n_{\mathrm{e}}$ variation, due to both the low excitation and ionization from the $2^{3} S$ state. Figure 10 shows the contributions of the two metastables due to $T_{\mathrm{e}}$ fluctuation calculated also at frequency $10^{3} \mathrm{~Hz}$. In this case, the $2^{1} S$ contribution is relatively large for the He I $667.8 \mathrm{~nm}$ line at $T_{\mathrm{e}} \lesssim 30 \mathrm{eV}(\sim 10 \%-20 \%)$, but still very small for the other two spectral lines $(<5 \%)$. The $2^{3} S$ contribution is $\sim 10 \%-50 \%$ for the $\mathrm{He}$ I $667.8 \mathrm{~nm}$ line at $T_{\mathrm{e}} \lesssim 20 \mathrm{eV}$ and significantly large for the He I $706.5 \mathrm{~nm}$ line. Both metastable contributions to the He I $728.1 \mathrm{~nm}$ line are not important. Some regions where the temperature related fluctuation is close to zero (see Fig. 7) should not be considered.

The strong dependence of the triplet level population on the $2^{3} S$ metastable has been previously confirmed by many studies. ${ }^{22-24}$ It is generally considered that the $2^{3} S$ state has a much higher population than the $2^{1} S$ metastable because a large portion of the population de-excited from the triplet levels to the $2^{3} S$ state is quickly and predominantly reexcited back. ${ }^{25}$ Thus, the population contributed from the $2^{3} S$ state to the triplet levels is relatively large and the population fluctuation in the triplet level is also significantly affected. Unfortunately, some experiments indicate that under certain plasma conditions the $2^{3} S$ population should be much lower than the value estimated by the quasi-static approximation. ${ }^{23,24}$ This leads to a large uncertainty by using spectral lines emitted from the triplet levels for the characterization of plasma parameters. Meanwhile, we notice that the He I $728.1 \mathrm{~nm}$ line is almost not affected by either of the two metastables. Therefore, this atomic line will be good for the plasma fluctuation measurements, particularly suitable for determining $n_{\mathrm{e}}$ fluctuation since it is not sensitive to $T_{\mathrm{e}}$ fluctuation at relatively high temperatures $\left(T_{\mathrm{e}} \gtrsim 50 \mathrm{eV}\right.$. Also, the insensitivity of the He I $728.1 \mathrm{~nm}$ line to the metastables results the independence of the fluctuation ratio on frequency, which is another merit for plasma fluctuation measurements. It is noteworthy that for statistic techniques such as correlation lengths and times and flow velocities ${ }^{11-13}$ that are commonly used for quantifying plasma fluctuations from light fluctuation data, the influences of the metastables are negligible. These statistic techniques are only dependent on the relative intensities of the measured light fluctuation signals, thus are almost not affected by the different dependencies of the line emission on $n_{\mathrm{e}}$ and $T_{\mathrm{e}}$, as discussed in Refs. 11,12 . However, these measured quantities are not necessarily the same as those of the underlying plasma fluctuations, due to the complicated relationship between the light signal and the local plasma parameters.

\section{NUMERICAL TESTS AND MEASUREMENT}

The relations between fluctuations of the atomic line emission and the underlying $n_{\mathrm{e}}$ and $T_{\mathrm{e}}$, which were obtained

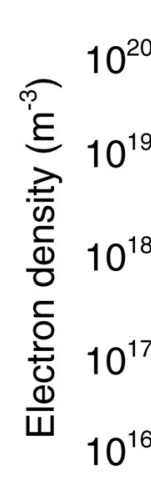

$2^{1} s$

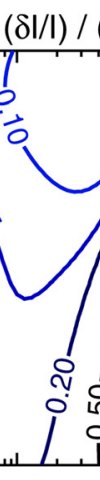

$\left(\delta T_{e} / T_{e}\right) 667$

667.8

10
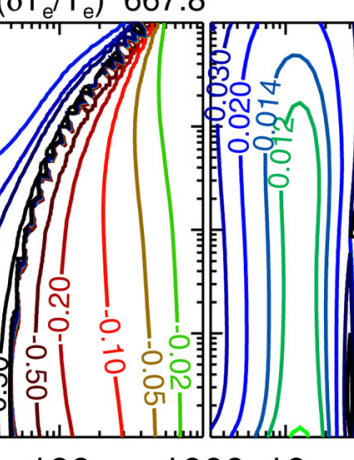

706.5

(a)

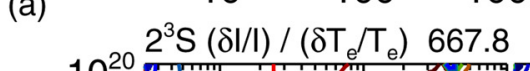

1000
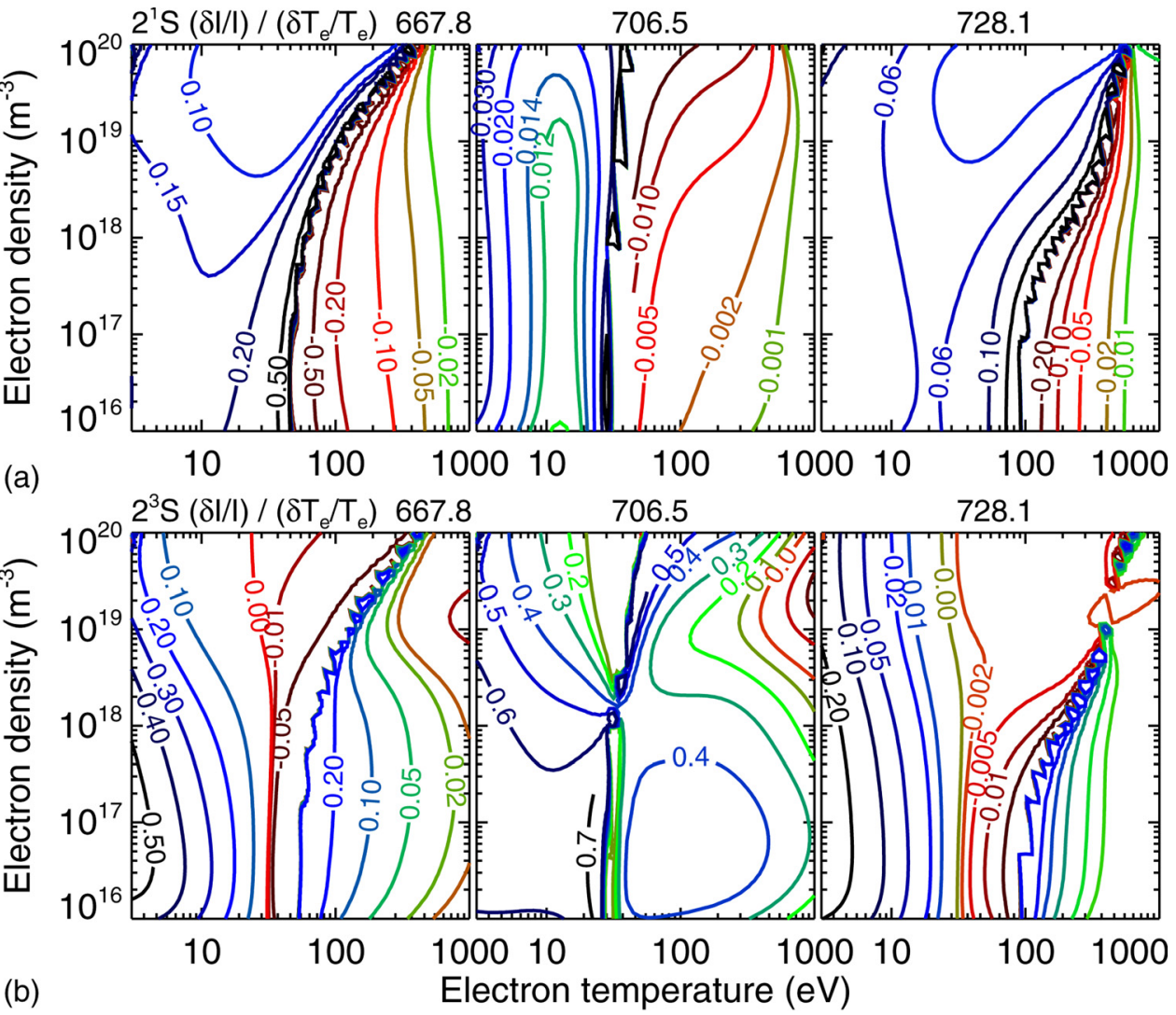

728.1
FIG. 10. (Color online) Contributions of the (a) $2^{1} S$ and (b) $2^{3} S$ metastable states population fluctuations to the three neutral helium line emission fluctuations in the $T_{\mathrm{e}}$ fluctuation related component at $10^{3} \mathrm{~Hz}$. 
with the linear approximation algorithm described in Sec. III, were numerically tested by using the time dependent CR model with artificial fluctuation data for the $n_{\mathrm{e}}$ and $T_{\mathrm{e}}$ fluctuations. The property of the artificial plasma fluctuation signals was set to about the same values measured by experiments. ${ }^{8}$ A broadband amplitude distribution (1-400 $\mathrm{kHz}$ ) with a decaying exponential tail in frequency space together with random phases was used to generate the fluctuation signals for $n_{\mathrm{e}}$ and $T_{\mathrm{e}}$ by inverse Fourier transform. Figure 11 shows the generated $n_{\mathrm{e}}$ and $T_{\mathrm{e}}$ fluctuation signals with $2 \times 10^{5}$ elements in $1 \mathrm{~ms}$ time interval and their frequency fluctuation amplitude distributions. The relative fluctuation level is $\sim 2 \%$ for both $n_{\mathrm{e}}$ and $T_{\mathrm{e}}$, and the time resolution is $5 \mathrm{~ns}$. It is important to note that the $n_{\mathrm{e}}$ and $T_{\mathrm{e}}$ fluctuations in Ref. 8 were measured in the core plasma region, whereas most optical methods such as beam emission spectroscopy and gas puff imaging can only access the plasma edge region. The plasma fluctuations in the edge region are usually very large compared with those in the core region (see Ref. 1 for more details). Since the model in this paper is zero-dimensional, it cannot discern any differences in the core and edge regions except the ground state density and the $n_{\mathrm{e}}$ and $T_{\mathrm{e}}$. Therefore, using the relatively low fluctuation data measured in the plasma core region is more suitable to test the accuracy of the linear approximation algorithm.

With the numerical fluctuation signals being imposed on the values of $n_{\mathrm{e}}$ or $T_{\mathrm{e}}$, respectively, as the input to the time dependent CR model and with the quasi-static population densities of the excited levels as the initial condition, and keeping other plasma parameters to be constants, namely, $T_{\mathrm{e}}=50 \mathrm{eV}$ (for density fluctuation) and $n_{\mathrm{e}}=10^{18} \mathrm{~m}^{-3}$ (for temperature fluctuation), the variations of the population densities in all excited levels as functions of time were calculated, and then the emission fluctuations for the three neutral helium lines (He I 667.8, 706.5, and 728.1 nm) were deduced
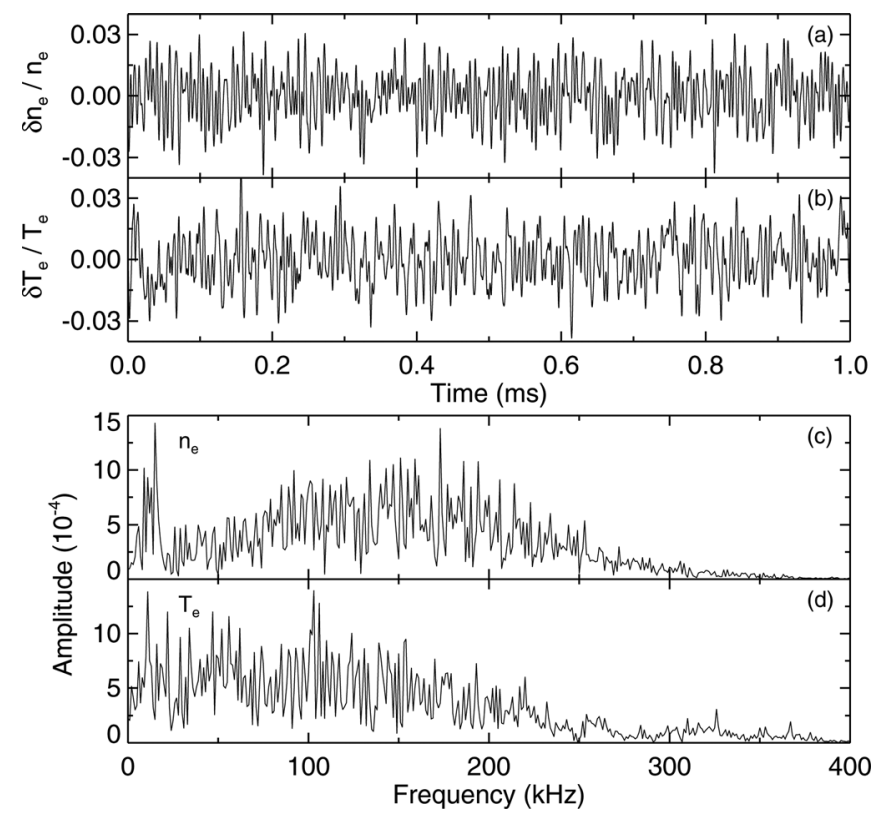

FIG. 11. Numerically generated relative fluctuation signals for (a) $n_{\mathrm{e}}$ and (b) $T_{\mathrm{e}}$ and $(\mathrm{c}, \mathrm{d})$ the corresponding frequency fluctuation amplitude distributions.
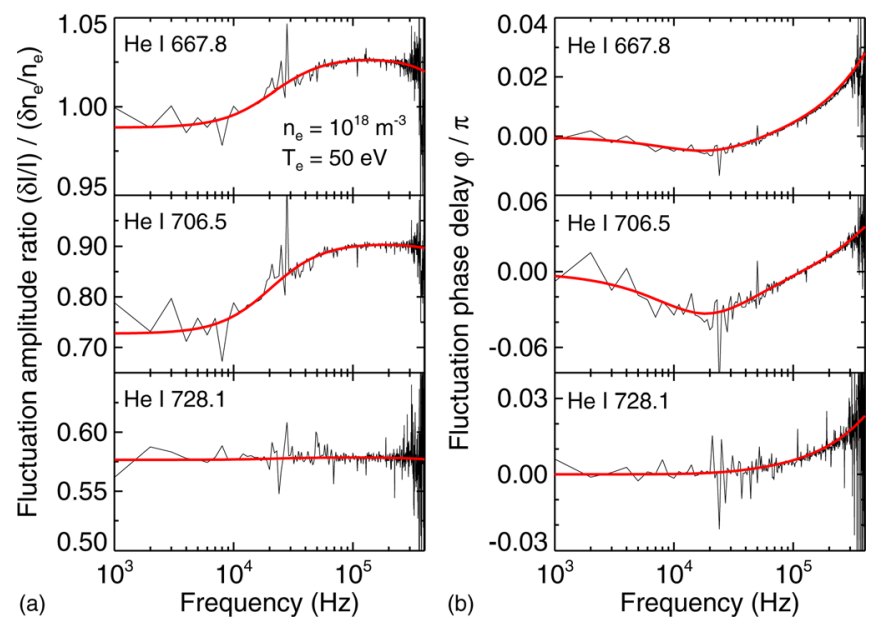

FIG. 12. (Color online) Comparison of (a) fluctuation ratio, $(\delta I / I) /\left(\delta n_{\mathrm{e}} / n_{\mathrm{e}}\right)$, and (b) phase delay, $\varphi / \pi$, due to $n_{\mathrm{e}}$ fluctuations as a function of frequency calculated from the time dependent CR model (thin line) and from the linear approximation technique (thick line) for the three helium lines with $n_{\mathrm{e}}=10^{18} \mathrm{~m}^{-3}$ and $T_{\mathrm{e}}=50 \mathrm{eV}$.

from Eq. (13). The results shown in Figs. 12 and 13 are the line emission fluctuation amplitudes and phase delays as functions of frequency due to, respectively, $n_{\mathrm{e}}$ and $T_{\mathrm{e}}$ fluctuations. These frequency dependent profiles were obtained by directly applying the fast Fourier transform to the line emission fluctuation signals without any further processing. The emission fluctuation amplitudes of the three helium lines are normalized respect to the fluctuation amplitudes of $n_{\mathrm{e}}$ or $T_{\mathrm{e}}$. The values obtained by the linear approximation algorithm are also included in each graph for a comparison. One can clearly see that the results obtained from both of the methods are in good agreement with each other over the whole frequency range, noticing that the spikes around $20-30 \mathrm{kHz}$ are due to numerical errors because the $n_{\mathrm{e}}$ and $T_{\mathrm{e}}$ fluctuation amplitudes are close to zero near the above frequencies [see Figs. 11(c) and 11(d)]. The consistent results validate the linear approximation algorithm, and also indicate that
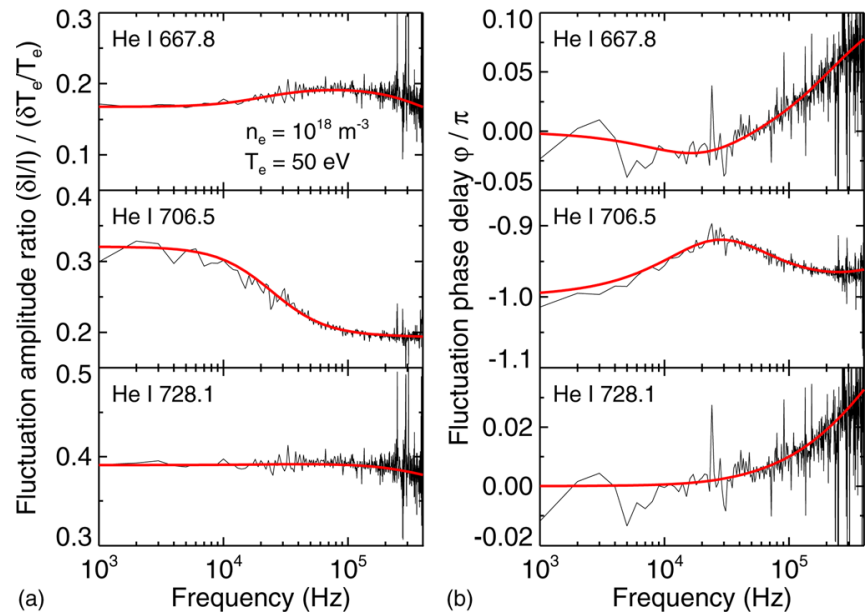

FIG. 13. (Color online) Comparison of (a) fluctuation ratio, $(\delta I / I) /\left(\delta T_{\mathrm{e}} / T_{\mathrm{e}}\right)$, and (b) phase delay, $\varphi / \pi$, due to $T_{\mathrm{e}}$ fluctuations as a function of frequency calculated from the time dependent CR model (thin line) and from the linear approximation technique (thick line) for the three helium lines with $n_{\mathrm{e}}=10^{18} \mathrm{~m}^{-3}$ and $T_{\mathrm{e}}=50 \mathrm{eV}$. 
recovering $n_{\mathrm{e}}$ or $T_{\mathrm{e}}$ fluctuations from the fluctuating signals of spectral line emissions is possible, provided all important physical processes are included in the model.

In the literature, many experiments have shown strong correlations between light emissions and Langmuir probe measured fluctuations, such as in Caltech, ${ }^{14}$ CSDX,${ }^{38}$ TPE$\mathrm{RX},{ }^{39}$ and Mirabelle. ${ }^{37}$ If the temperature related fluctuation is negligible (as shown in Sec. IV C for neutral helium, for other gases the results should be in a similar situation) or in phase with the $n_{\mathrm{e}}$ fluctuation (as sometimes measured by means of Langmuir probes in TEXT-U (Ref. 40) and DIII-D (Ref. 41) and expected theoretically, see Ref. 11), the interpretation of the observed line emission signals will be largely simplified. Currently, the two-dimensional optical measurement of $n_{\mathrm{e}}$ and $T_{\mathrm{e}}$ fluctuations in the $\mathrm{H}-1$ heliac $^{42}$ is still in progress. The helium plasma produced in the $\mathrm{H}-1$ device has $T_{\mathrm{e}}$ in the range $20-30 \mathrm{eV}$ and $n_{\mathrm{e}} \sim 10^{18} \mathrm{~m}^{-3}$. The dominant coherent fluctuations within the plasma have frequency in the range $15-20 \mathrm{kHz}$ and fluctuation levels below $10 \%$, as studied using virous Langmuir probes. ${ }^{43}$ Based on the experiment, we expect to determine the values of $n_{\mathrm{e}}$ and $T_{\mathrm{e}}$ in the plasma by the helium line intensity ratio method and then recover the $n_{\mathrm{e}}$ and $T_{\mathrm{e}}$ fluctuations with the relationship presented in this paper.

\section{DISCUSSION}

The relations between the emission of the three neutral helium lines (He I 667.8, 706.5, and $728.1 \mathrm{~nm}$ ) and the underlying $n_{\mathrm{e}}$ and $T_{\mathrm{e}}$ fluctuations were studied mainly based on the assumptions that the fluctuation level within the plasma is not very high and the ground state density fluctuation is negligible. For the application of the calculated results to interpret optical observations, it is important to know under what conditions these assumptions are valid and the possible effects of different plasma parameters. We discuss these issues in this section.

\section{A. Linear $\mathrm{n}_{\mathrm{e}}$ and $\mathrm{T}_{\mathrm{e}}$ fluctuation dependencies of line emission fluctuations}

The results obtained by the linear approximation technique assume that the line emission fluctuation is linearly dependent on $n_{\mathrm{e}}$ and $T_{\mathrm{e}}$ fluctuations. If the linear relationship is not satisfied, the line emission fluctuation should also be a function of the $n_{\mathrm{e}}$ and $T_{\mathrm{e}}$ fluctuations. The dependence of the line emission on plasma fluctuation levels will complicate the interpretation of experimentally observed light signals. It is therefore necessary to check to what extent the linear relationship is still valid.

By changing the harmonic fluctuation levels of $n_{\mathrm{e}}$ and $T_{\mathrm{e}}$ in the time dependent CR model, the fluctuation amplitude ratio of the line emission to the density or temperature at each frequency was obtained. Figures 14(a) and 14(b) show the ratios for $n_{\mathrm{e}}$ and $T_{\mathrm{e}}$, respectively, with several fluctuation levels from 0.1 to 0.5 and those from the linear approximation algorithm, calculated with $n_{\mathrm{e}}=5 \times 10^{17} \mathrm{~m}^{-3}$ and $T_{\mathrm{e}}=50 \mathrm{eV}$. Comparing the results from the two methods, it is easy to see that for fluctuation levels up to 0.1 the fluctuation of the line emission is still linearly dependent on the

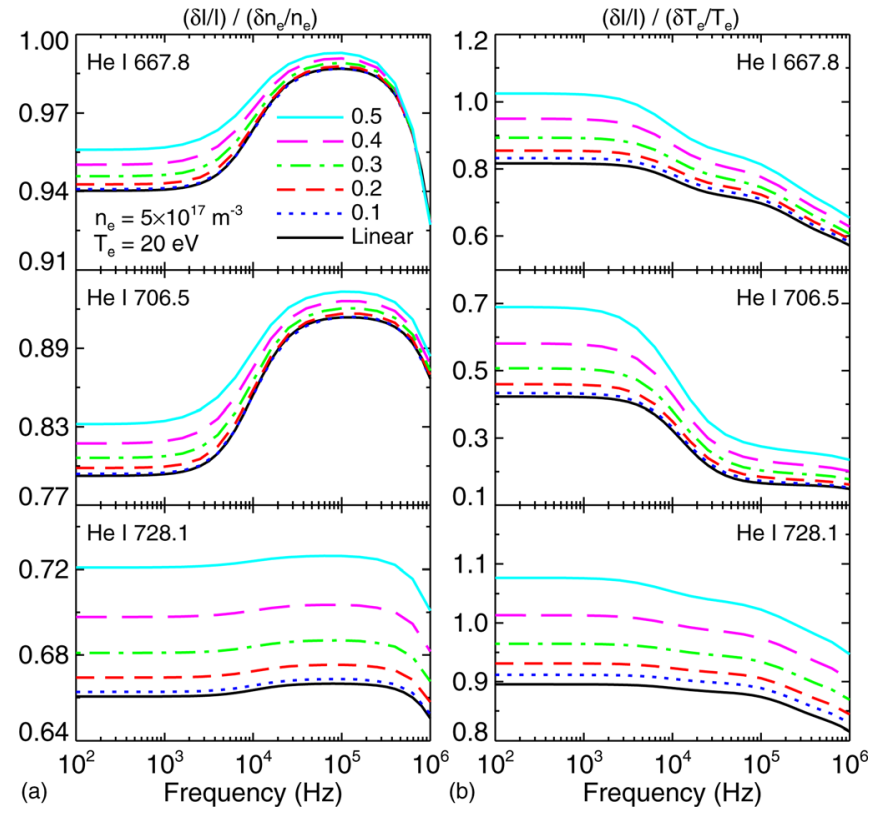

FIG. 14. (Color online) Comparison of the fluctuation amplitude ratios calculated by the linear approximation technique and the time dependent CR model with fluctuation levels of 0.1 to 0.5 for (a) $n_{\mathrm{e}}$ and (b) $T_{\mathrm{e}}$ related fluctuations. $n_{\mathrm{e}}=5 \times 10^{17} \mathrm{~m}^{-3}$ and $T_{\mathrm{e}}=20 \mathrm{eV}$.

fluctuations of $n_{\mathrm{e}}$ and $T_{\mathrm{e}}$. Even with the large fluctuation level up to 0.3 , the deviations from the linear relationship for the three helium lines are only $0.6 \%, 2 \%, 3 \%$ and $9 \%, 20 \%$, $8 \%$, respectively, for the $n_{\mathrm{e}}$ and $T_{\mathrm{e}}$ fluctuations. The much higher deviation for the He I $706.5 \mathrm{~nm}$ line in the case of $T_{\mathrm{e}}$ fluctuation is due to the fluctuation insensitivity of the line with the current plasma parameters (see Fig. 7). The larger deviation for $T_{\mathrm{e}}$ fluctuation compared with that for $n_{\mathrm{e}}$ fluctuation can be explained based on the profiles shown in Fig. 3, because the line emission is less linearly dependent on $T_{\mathrm{e}}$ than on $n_{\mathrm{e}}$. From the profiles in Fig. 3 we can also see that for temperatures lower or higher than $\sim 20 \mathrm{eV}$ the line emission can be better approximated as the linear $T_{\mathrm{e}}$ dependence, thus the linearity should be held to a larger fluctuation level for other plasma parameters.

\section{B. Fluctuation sensitivity on plasma parameters}

The line intensity ratio method is sensitive to atomic data since it is dependent on both of the line intensities in the line ratio pair. In contrast, the relationship between the light emission fluctuation and the $n_{\mathrm{e}}$ and $T_{\mathrm{e}}$ fluctuations only depends on the slope of the line emission profiles as a function of $n_{\mathrm{e}}$ and $T_{\mathrm{e}}$ and is, thus, not sensitive to atomic data. The line emission fluctuation is also not sensitive to the values of $n_{\mathrm{e}}$ and $T_{\mathrm{e}}$, as shown in Figs. 5 and 7. The insensitivity to plasma parameters thus does not require the accurate determination of $n_{\mathrm{e}}$ and $T_{\mathrm{e}}$ within the plasma. The ion density fluctuation is found to be not important, due to the fact that the contribution of the ion to the excited level population is small under the ionizing plasma condition. If the ground state density is constant, the line emission fluctuation will be independent of the ground state density, because for ionizing plasmas the population ratio of the excited level to the ground state is only a function of $n_{\mathrm{e}}$ and $T_{\mathrm{e}}$, as described by 
Eq. (13). The metastables may have some effects on the line emission fluctuations. The population of the $2^{3} S$ metastable is possibly not accurately described by the quasi-static approximation as suggested in some experiments. ${ }^{23,24}$ Hence, there is a large uncertainty for the spectral lines emitted from the triplet levels. On the other hand, the singlet lines are not sensitive to the metastable populations and thus are less likely to be affected, as discussed in Sec. IV D.

In the fluctuation model, the main uncertainty is the assumption for the ground state density which is considered to be without fluctuation. We find that if the variation of the ground state density is dominated by the ionization process the line emission fluctuation property will be affected. This influence, however, is limited only to frequencies lower than the value corresponding to the ionization time constant of the ground state. In Fig. 2(b) we presented the ground state ionization time with different plasma parameters. The results show that at relatively low $n_{\mathrm{e}}$ or $T_{\mathrm{e}}$ values the ionization time is long, thus under these plasma conditions the influence of the ground state density fluctuation is not important for high frequency fluctuations. In the case of gas puff imaging, a two-dimensional simulation ${ }^{44}$ shows that the possible effect of the neutral density fluctuation caused by plasma turbulence (the "shadowing" effect ${ }^{44}$ ) is not negligible compared with fluctuations in $n_{\mathrm{e}}$ and $T_{\mathrm{e}}$. But in experiments ${ }^{11}$ little or no sign indicating the fluctuation of the neutral density was observed. Notice that in the simulation the plasma perturbation was highly idealized and may differ significantly from the experimental condition. The possible "shadowing" effect which is not noticeable in the experiments is perhaps due to the difficulties in isolating this phenomenon experimentally or due to the averaging of the neutral density over the plasma turbulence structures, both temporally and spatially. ${ }^{34}$ If the averaging effect is comparable or more important than the ionization process, the ground state density is less possibly being changed by the ionization process or at least with a variation not as significant as predicted by the ionization process described by Eq. (6). Nevertheless, the possible effect of the ground state density fluctuation should be evaluated both experimentally and theoretically to determine under what conditions it is not important.

\section{CONCLUSIONS}

On the basis of a CR model for neutral helium together with a linear approximation technique, the relations between the emission fluctuation of three helium lines (He I 667.8, 706.5 , and $728.1 \mathrm{~nm}$ ) and the underlying $n_{\mathrm{e}}$ and $T_{\mathrm{e}}$ fluctuations, which are important for the quantitative interpretation of optical measurements of fusion plasma fluctuations, were investigated in detail. The relative fluctuation amplitude ratios between the line emission and the $n_{\mathrm{e}}$ and $T_{\mathrm{e}}$ are almost constant and the phase delays are close to zero or $-\pi$ for a wide range of practically interested frequencies $(<1 \mathrm{MHz})$. The line emissions become less sensitive to density fluctuations with increasing $n_{\mathrm{e}}$ and have little sensitivity to temperature fluctuations for relatively high $T_{\mathrm{e}}$ values. These results are in good agreement with the quasi-static estimations under low frequency fluctuations. When the fluctuation frequency is higher than the value corresponding to the relaxation time of the upper transition state of the spectral line, the ratio sharply decreases and the phase delay increases, indicating that there is no response to the plasma fluctuations. Numerical simulations done by using synthesized $n_{\mathrm{e}}$ and $T_{\mathrm{e}}$ fluctuation signals as the inputs to the time dependent $\mathrm{CR}$ model generated consistent fluctuation ratio and phase delay profiles compared with those from the linear approximation technique, suggesting both $n_{\mathrm{e}}$ and $T_{\mathrm{e}}$ fluctuations can be recovered from light emissions, given that the relationship between the line emission and the plasma fluctuations are provided by a CR model.

Since the linear approximation technique was used, the results are only applicable for plasma fluctuation levels up to $\sim 10 \%$ under which the linear relationship between fluctuations of the line emission and the $n_{\mathrm{e}}$ and $T_{\mathrm{e}}$ is still valid. Fortunately, the linearity does not deviate too much even for fluctuation levels up to $50 \%$. One of the possible uncertainty in the model is the assumption that the $2^{1} S$ and $2^{3} S$ metastable states of neutral helium are in equilibrium with other energy levels, which is questionable for plasmas under certain conditions. In most cases, the $2^{1} S$ contribution is negligible while the $2^{3} S$ contribution is important for the triplet spectral lines (e.g., the He I $706.5 \mathrm{~nm}$ line). The He I 728.1 $\mathrm{nm}$ line emitted from the singlet level is not sensitive to either of the metastables and has a very small response to $T_{\mathrm{e}}$ fluctuation at a large range of temperatures it is thus suitable for $n_{\mathrm{e}}$ fluctuation measurements. Another uncertainty in the model is the influence of the ground state density fluctuation. This fluctuation if exists, it will affect the line emission for frequencies lower than the value corresponding to the ionization time of the ground state. However, such fluctuation was not observed in gas puff imaging experiments perhaps due to difficulties in experiments to detect the phenomenon or due to factors such as the averaging over turbulence structures which will at least partly compensate the ionization effect.

In summary, we investigated the frequency dependent relations between the helium line emission fluctuations and the underlying $n_{\mathrm{e}}$ and $T_{\mathrm{e}}$ fluctuations by a neutral helium CR model for the interpretation of plasma fluctuations. The limitation of the model is that the linear relationship between fluctuations in the line emission and in $n_{\mathrm{e}}$ and $T_{\mathrm{e}}$ is valid for plasma fluctuation levels up to only $\sim 10 \%$, and the possible uncertainties are the effects of the triplet metastable and the ground state density fluctuation. To further investigate these problems, both experiments and simulations are needed.

\section{ACKNOWLEDGMENTS}

The authors gratefully acknowledge M. Goto (National Institute of Fusion Science) for providing the $\mathrm{CR}$ model code. One of the authors (S. Ma) thanks D. P. Stotler, S. J. Zweben (Princeton Plasma Physics Laboratory) and M. Agostini (Consorzio RFX) for helpful comments. The authors also thank the referee for many valuable remarks and suggestions which made this article more readable.

${ }^{1}$ J. D. Callen, Phys. Fluids B 4, 2142 (1992).

${ }^{2}$ A. J. Wootton, B. A. Carreras, H. Matsumoto, K. McGuire, W. A. Peebles, Ch. P. Ritz, P. W. Terry, and S. J. Zweben, Phys. Fluids B 2, 2879 (1990). 
${ }^{3}$ N. Bretz, Rev. Sci. Instrum. 68, 2927 (1997).

${ }^{4}$ S. J. Zweben, J. A. Boedo, O. Grulke, C. Hidalgo, B. LaBombard, R. J. Maqueda, P. Scarin, and J. L. Terry, Plasma Phys. Control. Fusion 49, S1 (2007).

${ }^{5}$ H. Evensen, D. Brouchous, D. Diebold, M. Doczy, R. J. Fonck, and D. Nolan, Rev. Sci. Instrum. 63, 4928 (1992).

${ }^{6}$ R. D. Durst, R. J. Fonck, G. Cosby, H. Evensen, and S. F. Paul, Rev. Sci. Instrum. 63, 4907 (1992).

${ }^{7}$ G. R. McKee, C. Fenzi, R. J. Fonck, and M. Jakubowski, Rev. Sci. Instrum. 74, 2014 (2003).

${ }^{8}$ A. E. White, L. Schmitz, G. R. McKee, C. Holland, W. A. Peebles, T. A. Carter, M. W. Shafer, M. E. Austin, K. H. Burrell, J. Candy, J. C. DeBoo, E. J. Doyle, M. A. Makowski, R. Prater, T. L. Rhodes, G. M. Staebler, G. R. Tynan, R. E. Waltz, and G. Wang, Phys. Plasmas 15, 056116 (2008).

${ }^{9}$ S. J. Zweben, D. P. Stotler, J. L. Terry, B. LaBombard, M. Greenwald, M. Muterspaugh, C. S. Pitcher, the Alcator C-Mod Group, K. Hallatschek, R. J. Maqueda, B. Rogers, J. L. Lowrance, V. J. Mastrocola, and G. F. Renda, Phys. Plasmas 9, 1981 (2002).

${ }^{10}$ R. J. Maqueda, G. A. Wurden, D. P. Stotler, S. J. Zweben, B. LaBombard, J. L. Terry, J. L. Lowrance, V. J. Mastrocola, G. F. Renda, D. A. D'Ippolito, J. R. Myra, and N. Nishino, Rev. Sci. Instrum. 74, 2020 (2003).

${ }^{11}$ S. J. Zweben, R. J. Maqueda, D. P. Stotler, A. Keesee, J. Boedo, C. E. Bush, S. M. Kaye, B. LeBlanc, J. L. Lowrance, V. J. Mastrocola, R. Maingi, N. Nishino, G. Renda, D. W. Swain, J. B. Wilgen, and the NSTX Team, Nucl. Fusion 44, 134 (2004).

${ }^{12}$ S. J. Zweben, R. J. Maqueda, R. Hager, K. Hallatschek, S. M. Kaye, T. Munsat, F. M. Poli, A. L. Roquemore, Y. Sechrest, and D. P. Stotler, Phys. Plasmas 17, 102502 (2010).

${ }^{13}$ Y. Sechrest, T. Munsat, D. A. D'Ippolito, R. J. Maqueda, J. R. Myra, D. Russell, and S. J. Zweben, Phys. Plasmas 18, 012502 (2011).

${ }^{14}$ S. J. Zweben, J. McChesney, and R. W. Gould, Nucl. Fusion 23, 825 (1983).

${ }^{15}$ M. Endler, H. Niedermeyer, L. Giannone, E. Holzhauer, A. Rudyj, G. Theimer, N. Tsois, and the ASDEX Team, Nucl. Fusion 35, 1307 (1995).

${ }^{16}$ Y. Ralchenko, R. K. Janev, T. Kato, D. V. Fursa, I. Bray, and F. J. de Heer, At. Data Nucl. Data Tables 94, 603 (2008).

${ }^{17}$ S. Sasaki, S. Takamura, S. Watanabe, S. Masuzaki, T. Kato, and K. Kadota, Rev. Sci. Instrum. 67, 3521 (1996).

${ }^{18}$ T. Nakano, H. Kubo, and N. Asakura, J. Phys. B: At. Mol. Opt. Phys. 43, 144014 (2010).

${ }^{19}$ S. Kajita, N. Ohno, S. Takamura, and T. Nakano, Phys. Plasmas 13, 013301 (2006); Erratum: 16(2), 029901 (2009).

${ }^{20}$ D. Nishijima and E. M. Hollmann, Plasma Phys. Controlled Fusion 49, 791 (2007).
${ }^{21}$ O. Schmitz, I. L. Beigman, L. A. Vainshtein, B. Schweer, M. Kantor, A. Pospieszczyk, Y. Xu, M. Krychowiak, M. Lehnen, U. Samm, B. Unterberg, and the TEXTOR team, Plasma Phys. Controlled Fusion 50, 115004 (2008).

${ }^{22}$ J-W. Ahn, D. Craig, G. Fiksel, D. J. Den Hartog, J. K. Anderson, and M. O'Mullane, Phys. Plasmas 14, 083301 (2007).

${ }^{23}$ R. Prakash, P. Vasu, V. Kumar, R. Manchanda, M. B. Chowdhuri, and M. Goto, J. Appl. Phys. 97, 043301 (2005).

${ }^{24}$ R. F. Boivin, S. D. Loch, C. P. Ballance, D. Branscomb, and M. S. Pindzola, Plasma Sources Sci. Technol. 16, 470 (2007).

${ }^{25}$ R. F. Boivin, J. L. Kline, and E. E. Scime, Phys. Plasmas 8, 5303 (2001).

${ }^{26}$ M. Goto, K. Sawada, and T. Fujimoto, Phys. Plasmas 9, 4316 (2002).

${ }^{27}$ T. Fujimoto, Plasma Spectroscopy (Clarendon, Oxford, 2004).

${ }^{28}$ T. Fujimoto, J. Quant. Spectrosc. Radiat. Transf. 21, 439 (1979).

${ }^{29}$ M. Goto, J. Quant. Spectrosc. Radiat. Transf. 76, 331 (2003).

${ }^{30}$ D. R. Bates, A. E. Kingston, and R. W. P. McWhirter, Pro. Roy. Soc. London 267, 297 (1962).

${ }^{31}$ L. C. Johnson and E. Hinnov, J. Quant. Spectrosc. Radiat. Transf. 13, 333 (1973).

${ }^{32}$ H. P. Summers, W. J. Dickson, M. G. O’Mullane, N. R. Badnell, A. D. Whiteford, D. H. Brooks, J. Lang, S. D. Loch, and D. C. Griffin, Plasma Phys. Controlled Fusion 48, 263 (2006).

${ }^{33}$ P. T. Greenland, Proc. R. Soc. London 457, 1821 (2001).

${ }^{34}$ D. P. Stotler, J. Boedo, B. LeBlanc, R. J. Maqueda, and S. J. Zweben, J. Nucl. Mater. 363-365, 686 (2007).

${ }^{35}$ J. Abdallah Jr., N. Palmer, W. Gekelman, J. Maggs, and R. E. H. Clark, J. Phys. B 32, 1001 (1999).

${ }^{36}$ M. S. Hegde and P. K. Ghosh, Pramana 12, 367 (1979).

${ }^{37}$ S. Oldenbürger, C. Brandt, F. Brochard, N. Lemoine, and G. Bonhomme, Rev. Sci. Instrum. 81, 063505 (2010).

${ }^{38}$ G. Y. Antar, J. H. Yu, and G. Tynan, Phys. Plasmas 14, 022301 (2007).

${ }^{39}$ M. Agostini, R. Cavazzana, P. Scarin, G. Serianni, Y. Yagi, H. Koguchi, S. Kiyama, H. Sakakita, and Y. Hirano, Plasma Phys. Controlled Fusion 50, 095004 (2008).

${ }^{40}$ M. A. Meier, R. D. Bengtson, G. A. Hallock, and A. J. Wootton, Phys. Rev. Lett. 87, 085003 (2001).

${ }^{41}$ D. L. Rudakov, J. A. Boedo, R. A. Moyer, S. Krasheninnikov, A. W. Leonard, M. A. Mahdavi, G. R. McKee, G. D. Porter, P. C. Stangeby, J. G. Watkins, W. P. West, D. G. Whyte, and G. Antar, Plasma Phys. Controlled Fusion 44, 717 (2002).

${ }^{42}$ S. M. Hamberger, B. D. Blackwell, L. E. Sharp, and D. B. Shenton, Fusion Technol. 17, 123 (1990).

${ }^{43}$ M. G. Shats and J. Howard, Fusion Eng. Des. 34-35, 271 (1997).

${ }^{44}$ D. P. Stotler, B. LaBombard, J. L. Terry, and S. J. Zweben, J. Nucl. Mater. 313-316, 1066 (2003). 